EFI 06-20

hep-ph/0609195

September 2006

\title{
HADRON SPECTROSCOPY: THEORY AND EXPERIMENT
}

\author{
Jonathan L. Rosner \\ Enrico Fermi Institute and Department of Physics, University of Chicago \\ 5640 S. Ellis Avenue, Chicago IL 60637 USA
}

\begin{abstract}
Many new results on hadron spectra have been appearing in the past few years thanks to improved experimental techniques and searches in new channels. New theoretical techniques including refined methods of lattice QCD have kept pace with these developments. Much has been learned about states made of both light $(u, d$, and $s)$ and heavy $(c, b)$ quarks. The present review treats light-quark mesons, glueballs, hybrids, particles with a single $c$ or $b$ quark, charmonium $(c \bar{c})$, and bottomonium $(b \bar{b})$ states. Some prospects for further study are noted.
\end{abstract}

PACS numbers: 14.20.Lq, 14.40.Cs, 14.40.Gx, 14.40.Lb

\section{INTRODUCTION}

Quantum Chromodynamics (QCD) is our theory of the strong interactions: Its property of asymptotic freedom [1] allows one to interpret deep inelastic scattering experiments [2] in terms of pointlike nucleon constituents [3], and permits perturbative calculations of short-distance phenomena. However, we are far from understanding how it works at longer distances governing the spectra of hadrons. Many hadrons discovered recently have unexpected properties. We need to understand hadron spectra in order to separate electroweak physics from strong-interaction effects. Moreover, we may need to use our experience with QCD in dealing with any non-perturbative effects encountered at higher energies such as those to be probed by the Large Hadron Collider (LHC). The understanding of electroweak symmetry breaking may well require non-perturbative techniques at $\mathrm{TeV}$ scales similar to those useful for hadron spectroscopy at GeV scales. Sharpening spectroscopic techniques even may help understand the intricate structure of masses and transitions, itself the indication of a rich spectroscopy at the quark and lepton level.

The QCD scale is $\sim 200 \mathrm{MeV}$ (momentum) or $\sim 1 \mathrm{fm}$ (distance), where perturbation theory cannot be used. Although lattice gauge theories are, in principle, the way to describe effects in this regime, several other methods can provide information, especially for multi-quark and multi-hadron problems not yet feasible with lattice techniques. Some of these will be discussed briefly in Section 2, I will then describe some phenomena to which these methods can be applied. These include light-quark and no-quark mesons (Section 3), charmed (Section 4) and beauty/bottom (Section 5) hadrons, and heavy charmonium (Section 6) and bottomonium (Section 7). I 
conclude with some prospects for progress in the areas mentioned (Section 8) and summarize in Section 9. Baryon spectroscopy is the topic of a separate review in this issue [4]. An extended review of transitions in quarkonium, drawing on and extending some of the material in this article, may be found in Ref. [5].

\section{SOME THEORETICAL METHODS}

\subsection{Lattice gauge theories}

At momentum scales less than about $2 \mathrm{GeV} / c$ the QCD coupling constant $\alpha_{S}\left(Q^{2}\right)$ becomes large enough that perturbation theory cannot be used. [For recent compilations of evidence that $\alpha_{S}\left(Q^{2}\right)$ behaves as predicted by asymptotic freedom see Ref. [6].] Below this scale one must resort to non-perturbative methods to describe long-distance hadronic interactions.

If space-time is discretized, one can overcome the dependence in QCD on perturbation theory. Quark confinement is one consequence of this lattice gauge theory approach. An accurate description of the heavy quarkonium spectrum can be obtained once one takes account of degrees of freedom associated with the production of pairs of light $(u, d, s)$ quarks [7].

\subsection{Chiral dynamics, unitarity, and crossing symmetry}

The coupling of soft pions to hadronic systems is specified by PCAC (partial conservation of axial current) and current algebra [8]. When supplemented by constraints enforcing unitarity and crossing symmetry, current algebra becomes a powerful tool for describing the dynamics of mesons up to the GeV scale and baryons somewhat higher. An early application of the power of these principles to $\pi \pi$ scattering appears in Ref. 9]. Most treatments these days implement crossing symmetry using an elegant set of exact low-energy relations [10]. A systematic expansion in chiral loops is known as chiral perturbation theory and is described in detail in Refs. [11]. (For a modern review, see Ref. [12].)

Although chiral $L \times R$ symmetry is usually regarded as being spontaneously broken to the vector symmetry, with the symmetry breaking manifested through the existence of Nambu-Goldstone bosons like the pion, a Wigner-Weyl realization of the chiral symmetry would be visible through parity doubling of states. Some evidence for this in baryon spectra has been noted [13].

\subsection{Heavy quark symmetry}

Hadrons with one charmed or beauty quark can be regarded as "atoms" of QCD, with the light-quark and gluonic degrees of freedom playing the role of the electron(s) and the heavy quark playing the role of the nucleus. Properties of these systems tend to be very simple under the interchange $c \leftrightarrow b$, in the manner of isotope effects in nuclei. These features were first noted in Ref. [14] and systematically explored in Refs. [15]. One can combine heavy quark symmetry with chiral dynamics to predict the existence 
of a broken form of parity doubling in mesons containing a single heavy quark [16]. We will see that there is some evidence for this in charmed-strange mesons.

\subsection{Quark model potential descriptions}

Quarkonium states may be described as bound by a force whose short-distance behavior is approximately Coulombic (with a slow variation of coupling strength to account for asymptotic freedom) and whose long-distance behavior is linear to account for quark confinement [17]. (For early reviews see Refs. [18, 19, 20, 21]). One can estimate from such a potential (or a simpler interpolating version [22]) that a non-relativistic description for charmonium is quite crude, with characteristic velocities $\left\langle v^{2}\right\rangle \simeq 0.5$ for a charmed quark in a $c \bar{c}$ bound state. For a $b$ quark in a $b \bar{b}$ bound state $\left\langle v^{2}\right\rangle \simeq 0.15$, so the non-relativistic description begins to make some sense.

The partial widths for a ${ }^{3} S_{1}$ state to decay to a lepton pair through a virtual photon depend on the square of the relative wave function at the origin through the relation [23]

$$
\Gamma\left({ }^{3} S_{1} \rightarrow e^{+} e^{-}\right)=\frac{16 \pi \alpha^{2} e_{Q}^{2}|\Psi(0)|^{2}}{M^{2}}
$$

where $e_{Q}=2 / 3$ or $-1 / 3$ is the quark charge and $M$ is the mass of the ${ }^{3} S_{1}$ state. Thus leptonic partial widths probe the compactness of the quarkonium system, and provide important information complementary to level spacings. In a power-law potential $V(r) \sim \operatorname{sgn}(\nu) r^{\nu},|\Psi(0)|^{2}$ scales as $m_{Q}^{3 /(2+\nu)}$, or $\sim m_{Q}^{3}, m_{Q}^{3 / 2}, m_{Q}$ for $\nu=-1,0,1$ [20]. Thus the effective quark mass in a potential description is constrained by measured leptonic widths. In more fundamental descriptions such as lattice gauge theories similar constraints will hold.

Hyperfine and fine-structure splittings in quarkonium are sensitive to the Lorentz structure of the interquark interaction [18, 19, 24, 25]. Writing the effective potential $V(r)$ as the sum of Lorentz vector $V_{V}$ and Lorentz scalar $V_{S}$ contributions, one finds that the spin-spin interaction is due entirely to the Lorentz vector:

$$
V_{S S}(r)=\frac{\sigma_{1} \cdot \sigma_{2}}{6 m_{Q}^{2}} \nabla^{2} V_{V}(r)
$$

where $\sigma_{1}$ and $\sigma_{2}$ are Pauli matrices acting on the spins of the quark and antiquark, respectively. For a Coulomb-like potential $\sim-1 / r$ the Laplacian is proportional to $\delta^{3}(r)$, so that $V_{S S}(r)$ contributes to hyperfine splittings only for $\mathrm{S}$ waves, whose wave functions are non-zero at the origin. In QCD the coupling constant undergoes slow (logarithmic) variation with distance, leading to small non-zero contributions to hyperfine splittings for $L>0$ states.

Spin-orbit and tensor forces affect states with $L>0$. The spin-orbit potential is

$$
V_{L S}(r)=\frac{L \cdot S}{2 m_{Q}^{2} r}\left(3 \frac{d V_{V}}{d r}-\frac{d V_{S}}{d r}\right),
$$

where $L$ is the relative orbital angular momentum of $Q$ and $\bar{Q}$, while $S$ is the total quark spin. The tensor potential is

$$
V_{T}(r)=\frac{S_{12}}{12 m_{Q}^{2}}\left(\frac{1}{r} \frac{d V_{V}}{d r}-\frac{d^{2} V_{V}}{d r^{2}}\right),
$$


where $S_{12} \equiv 2\left[3(S \cdot \hat{r})(S \cdot \hat{r})-S^{2}\right]$ has non-zero expectation values only for $L>0$.

One must include relativistic effects to address the high quality of quarkonium data now available. Early treatments include Refs. [26] and [27] (with a recent application in [28]). The papers of Refs. [29] represent another area of effort in this regard. However, a recent review of relativistic corrections to electric dipole matrix elements [5] indicates that a satisfactory description of these properties of hadrons has not yet been reached.

Coupled-channel effects are also very important in heavy quarkonium spectroscopy, particularly for states near and above flavor threshold. The study of these was pioneered in Ref. [30] (for recent applications see [31]).

\subsection{Light quarks as quasi-particles}

One can reproduce a great deal of the spectrum of hadrons containing the $u, d$, and $s$ quarks with a simple model based on additive quark masses $m_{i}$ and hyperfine interactions proportional to $\left\langle\sigma_{i} \cdot \sigma_{j} /\left(m_{i} m_{j}\right)\right\rangle$ [32, 33, 34]. In this treatment the best fit to meson spectra occurs when $u$ and $d$ quarks have effective masses of $310 \mathrm{MeV} / c^{2}$ while $s$ quarks have effective masses of $485 \mathrm{MeV} / c^{2}$. The corresponding values for baryons are each shifted upward by $53 \mathrm{MeV} / c^{2}: 363$ and $538 \mathrm{MeV} / c^{2}$. These values are very different from the effective masses of quarks at $Q^{2}$ scales of $\left(2 \mathrm{GeV} / c^{2}\right)^{2}$, which are about 3, 6, and $100 \mathrm{MeV} / c^{2}$ [35]. The additional mass in the "constituent" quarks may be thought of as due to their interaction with the surrounding gluon field.

\subsection{Correlations among quarks}

Because the product of two color-SU(3) triplets consists of an antitriplet and a sextet, two color-triplet quarks can form a color antitriplet with spin zero or 1 . The spinspin force is attractive in the spin-zero state, so two non-identical quarks can form a spinless boson whose effective mass can be comparable to that of a single quark. An early realization that this fact implied a sort of supersymmetry in the hadron spectrum appears in Refs. [36]. Recent applications of this idea to hadron spectroscopy have appeared in Refs. [37, 38, 39]. Quantitative tests of these ideas include the prediction [37] of a weakly decaying $b q \bar{c} \bar{q}^{\prime}$ state.

\subsection{QCD sum rules and instantons}

Because of its increased coupling strength at long distances, QCD leads to the formation of condensates (similar to Cooper pairs in superconductivity), including non-zero expectation values of color singlet quark-antiquark pairs and gluonic configurations such as instantons. A systematic attempt to cope with the effect of these condensates on hadron spectroscopy relies on sum rules pioneered in Ref. [40]. For a more recent review of the role of instantons in hadron spectroscopy, see Ref. [41].

\subsection{Resonance decays}

An elegant treatment of hadronic resonance decays in which a single quark in a hadron undergoes pion emission was given in Ref. [42]. This work and similar treatments 
of electromagnetic decays [43, 44] are based on the work of Melosh [45] relating "current quarks" (those in the fundamental QCD Lagrangian) to "constituent quarks" (essentially quasi-particles in terms of which hadrons have simple properties).

From the standpoint of predictions the above single-quark-transitions is identical to a model in which resonance decays proceed via the creation of a ${ }^{3} P_{0}$ quarkantiquark pair with the quantum numbers of the vacuum, $J^{P C}=0^{++}$[46, 47, 48, 49]. In resonance decays involving two possible partial waves, these approaches involve independent amplitudes for each partial wave. Models based on explicit quark wave functions (e.g., [50, 51]) relate the partial waves to one another.

\section{LIGHT-QUARK STATES}

In this section we discuss a few topics of current interest in light-quark spectroscopy: the nature of the low-energy S-wave $\pi \pi$ and $K \pi$ interactions; the proliferation of interesting threshold effects in a variety of reactions, and the interaction of quark and gluonic degrees of freedom.

\subsection{Low-energy $\pi \pi$ S-wave}

An S-wave $\pi \pi$ low-mass correlation in the $I=0$ channel (" $\sigma$ ") has been used for many years to describe nuclear forces. Is it a resonance? What is its quark content? What can we learn about it from charm and beauty decays? This particle, otherwise known as $f_{0}(600)$ [35], can be described as a dynamical $I=J=0$ resonance in elastic $\pi \pi$ scattering using current algebra, crossing symmetry, and unitarity [9, 52, 53]. It appears as a pole with a large imaginary part with real part at or below $m_{\rho}$. Its effects differ in $\pi \pi \rightarrow \pi \pi$, where an Adler zero suppresses the low-energy amplitude, and inelastic processes such as $\gamma \gamma \rightarrow \pi \pi$ [54], where the lack of an Adler zero leads to larger contributions at low $m_{\pi \pi}$.

There is not unanimity regarding the exact position of the $\sigma$ pole. In one approach [55] it is found at $441-i 272 \mathrm{MeV}$, corresponding to a full width at half maximum of $544 \mathrm{MeV}$; another [56] finds it at $555-i 262 \mathrm{MeV}$. Such a $\sigma$ provides a good description of $\gamma \gamma \rightarrow \pi^{0} \pi^{0}$ [57], with $\Gamma(\sigma \rightarrow \gamma \gamma)=(4.1 \pm 0.3) \mathrm{keV}$. While this large partial width might be viewed as favoring a $q \bar{q}$ interpretation of $\sigma$ [57], a $\pi \pi$ dynamical resonance seems equally satisfactory [54]. Other recent manifestations of a $\sigma$ include the decays $D^{+} \rightarrow \sigma \pi^{+} \rightarrow \pi^{+} \pi^{-} \pi^{+}$[58] and $J / \psi \rightarrow \omega \sigma \rightarrow \omega \pi^{+} \pi^{-}$[59], where the $\sigma$ pole appears at $(541 \pm 39)-i(252 \pm 42) \mathrm{MeV}($ or $(500 \pm 30)-i(264 \pm 30) \mathrm{MeV}$ in an independent analysis [60]). Successful fits without a $\sigma$ have been performed, but have been criticized in Ref. 61].

\subsection{Low-energy $K \pi$ S-wave}

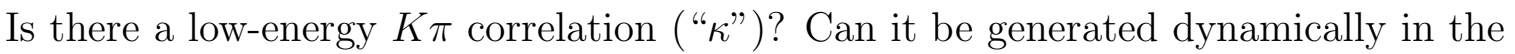
same manner as the $\sigma$ ? Some insights are provided in [53, 62].

The low-energy $K \pi$ interaction in the $I=1 / 2, J=0$ channel is favorable to dynamical resonance generation: The sign of the scattering length is the same as for 
the $I=J=0 \pi \pi$ interaction. A broad scalar resonance $\kappa$ is seen in the $I=1 / 2, J=$ $0 K^{-} \pi^{+}$subsystem in $D^{+} \rightarrow K^{-} \pi^{+} \pi^{+}$, and a model-independent phase shift analysis shows resonant $J=0$ behavior in this subsystem [63]. The $\kappa$ is also seen by the BES II Collaboration in $J / \psi \rightarrow \bar{K}^{* 0}(892) K^{+} \pi^{-}$decays [64. An independent analysis of the BES II data [56] finds a $\kappa$ pole at $745-i 316 \mathrm{MeV}$, while a combined analysis of $D^{+} \rightarrow K^{-} \pi^{+} \pi^{+}$, elastic $K \pi$ scattering, and the BES II data [65] finds a pole at $M(\kappa)=\left(750_{-55}^{+30}\right)-i(342 \pm 60) \mathrm{MeV}$.

The $\kappa$, like the $\sigma$, is optional in many descriptions of final-state interactions. For example, in a recent fit to the $D^{0} \rightarrow K^{+} K^{-} \pi^{0}$ Dalitz plot based on CLEO data [66], bands are seen corresponding to $K^{*-}, K^{*+}$, and $\phi$. One can see the effect of an S-wave amplitude interfering with $K^{*+}$ and $K^{*-}$ with opposite signs in the low- $K^{ \pm} \pi^{0}$-mass regions of the plot, but one cannot tell whether this amplitude is non-resonant or due to a $\kappa$. Depopulated regions at $m\left(K^{ \pm} \pi^{0}\right) \simeq 1 \mathrm{GeV} / c^{2}$ may be due to the opening of the $K \pi^{0} \rightarrow K \eta$ S-wave threshold (a $D^{0} \rightarrow K^{+} K^{-} \eta$ Dalitz plot would test this) or to a vanishing S-wave $K \pi$ amplitude between a $\kappa$ and a higher $J^{P}=0^{+}$resonance. A candidate for such a resonance exists around $1430 \mathrm{MeV} / \mathrm{c}^{2}$ [35].

\subsection{Dips and edges}

High-statistics Dalitz plots for heavy meson decays often exhibit dips and edges associated with thresholds [67. In a recent $D^{0} \rightarrow K_{S}^{0} \pi^{+} \pi^{-}$plot [68], sharp edges in the $\pi^{+} \pi^{-}$spectrum correspond to $\rho$ - $\omega$ interference [around $M(\pi \pi)=0.8 \mathrm{GeV} / c^{2}$ ] and to $\pi^{+} \pi^{-} \leftrightarrow K \bar{K}$ [around $M(\pi \pi)=1 \mathrm{GeV} / c^{2}$ ]. Rapid variation of an amplitude occurs when a new $\mathrm{S}$-wave channel opens because no centrifugal barrier is present.

Further dips are seen in $6 \pi$ photoproduction just at $p \bar{p}$ threshold; in $R_{e^{+} e^{-}}$just below the threshold for S-wave production of $D(1865)+D_{1}(2420)$; and in the Dalitz plot for $B^{ \pm} \rightarrow K^{ \pm} K^{\mp} K^{ \pm}$around $M\left(K^{+} K^{-}\right)=1.6 \mathrm{GeV} / c^{2}$ [69], which could be a threshold for vector meson pair production.

\subsection{Glueballs and hybrids}

In QCD, quarkless "glueballs" may be constructed from color-singlet polynomials in the field-strength tensor $F_{\mu \nu}^{a}$. All such states should be flavor-singlet with isospin $I=0$, though $s \bar{s}$ couplings of spinless states could be favored [70]. Lattice QCD predicts the lowest glueball to be $0^{++}$with $M \simeq 1.7 \mathrm{GeV}$ [71]. The next-lightest states, $2^{++}$and $0^{-+}$, are expected to be several hundred $\mathrm{MeV} / \mathrm{c}^{2}$ heavier. Thus it is reassuring that the lightest mainly flavor-singlet state, the $\eta^{\prime}$, is only gluonic $(14 \pm 4) \%$ of the time, as indicated by a recent measurement of $\mathcal{B}\left(\phi \rightarrow \eta^{\prime} \gamma\right)$ by the KLOE Collaboration [72].

Many other $I=0$ levels, e.g., $q \bar{q}, q \bar{q} g$ ( $g=$ gluon), $q q \bar{q} \bar{q}, \ldots$, can mix with glueballs. One must study $I=0$ levels and their mesonic couplings to separate out glueball, $n \bar{n} \equiv(u \bar{u}+d \bar{d}) / \sqrt{2}$, and $s \bar{s}$ components. Understanding the rest of the flavored $q \bar{q}$ spectrum for the same $J^{P}$ thus is crucial. The best $0^{++}$glueball candidates (mixing with $n \bar{n}$ and $s \bar{s}$ ) are at 1370,1500 , and $1700 \mathrm{MeV}$. One can explore their flavor structure through production and decay, including looking for their $\gamma(\rho, \omega, \phi)$ decays 
[73]. A CLEO search for such states in $\Upsilon(1 S) \rightarrow \gamma X$ finds no evidence for them but does see the familiar resonance $f_{2}(1270)$ [74.

QCD predicts that in addition to $q \bar{q}$ states there should be $q \bar{q} g$ ("hybrid") states containing a constituent gluon $g$. One signature of them would be states with quantum numbers forbidden for $q \bar{q}$ but allowed for $q \bar{q} g$. For $q \bar{q}, P=(-1)^{L+1}, C=$ $(-1)^{L+S}$, so $C P=(-1)^{S+1}$. The forbidden $q \bar{q}$ states are then those with $J^{P C}=0^{--}$ and $0^{+-}, 1^{-+}, 2^{+-}, \ldots$. In quenched lattice $\mathrm{QCD}$ most calculations find that the lightest exotic hybrids have $J^{P C}=1^{-+}$and $M(n \bar{n} g) \simeq 1.9 \mathrm{GeV}, M(s \bar{s} g) \simeq 2.1 \mathrm{GeV}$, with errors 0.1-0.2 GeV [75]. (Ref. [76] finds that such estimates, based on linear extrapolations to the chiral limit, may have overestimated these masses by as much as $0.3 \mathrm{GeV} / c^{2}$. Unquenched QCD must treat mixing with $q q \bar{q} \bar{q}$ and meson pairs.) Candidates for hybrids include $\pi_{1}(1400)$ (seen in some $\eta \pi$ final states, e.g., in $p \bar{p}$ annihilations) and $\pi_{1}(1600)$ (seen in $\left.3 \pi, \rho \pi, \eta^{\prime} \pi\right)$. While Brookhaven experiment E-852 published evidence for a $1^{-+}$state called $\pi_{1}(1600)$ [77], a recent analysis [78] does not require this particle if a $\pi_{2}(1670)$ contribution is assumed instead. The favored decays of a $1^{-+}$hybrid are to a $q \bar{q}(L=0)+q \bar{q}(L=1)$ pair, such as $\pi b_{1}(1235)$. A review of glueballs and hybrids has been presented recently [79], and lattice predictions for their decays have been discussed in Ref. [80].

\section{CHARMED STATES}

The present status of the lowest S-wave states with a single charmed quark is shown in Fig. 1. We will discuss progress on charmed baryons, on charmed-strange mesons, and on $D^{+}$and $D_{s}^{+}$decay constants.

\subsection{Charmed baryons}

The predicted spectroscopy of ground state baryons containing a single charmed quark was mapped out in 1974 [81]. At last, the family of predicted states has been completed with the report by the BaBar Collaboration of the $\Omega_{c}^{*}$ [82]. It is a candidate for the $J^{P}=3 / 2^{+}$state of the css system. It was found to lie $70.8 \pm 1.0 \pm 1.1 \mathrm{MeV} / c^{2}$ above the $\Omega_{c}$ (the candidate for the S-wave $J^{P}=1 / 2^{+}$css state), and to decay to $\gamma \Omega_{c}$. Its mass was as expected in models of QCD hyperfine splittings (e.g., [83]).

CLEO, BaBar, and Belle have contributed greatly to data on orbitally-excited charmed baryons, whose levels are plotted along with those of the lowest $L=0$ states in Fig. 2. The first excitations of the $\Lambda_{c}$ and $\Xi_{c}$ are similar, scaling well from the first $\Lambda$ excitations $\Lambda\left(1405,1 / 2^{-}\right)$and $\Lambda\left(1520,3 / 2^{-}\right)$. They have the same cost in $\Delta L$ (about $300 \mathrm{MeV}$ ), and their $L \cdot S$ splittings scale as $1 / m_{s}$ or $1 / m_{c}$. Higher $\Lambda_{c}$ states may correspond to excitation of a spin-zero $[u d]$ pair to $S=L=1$, leading to many allowed $J^{P}$ values up to $5 / 2^{-}$. In $\Sigma_{c}$ the light-quark pair has $S=1$; adding $L=1$ allows $J^{P} \leq 5 / 2^{-}$. States with higher $L$ may be narrower as a result of increased barrier factors affecting their decays, but genuine spin-parity analyses would be very valuable. Here are some recent results:

(1) The $\Lambda_{c}(2880)$ was first seen in the $\Lambda_{c}^{+} \pi^{-} \pi^{+}$mode by CLEO [84 and confirmed in the $D^{0} p$ mode recently by BaBar [85. It now has been shown to have likely 


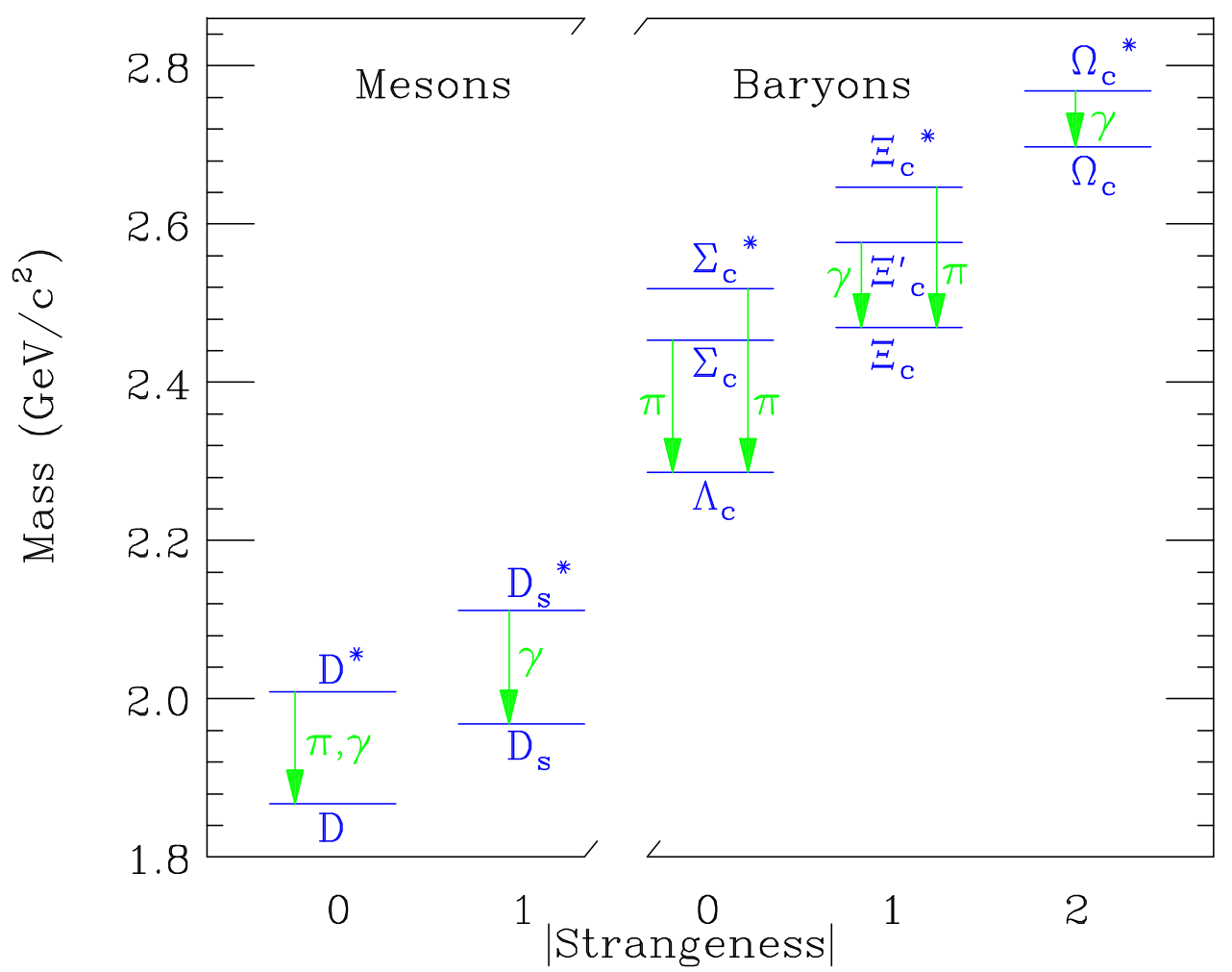

Figure 1: Lowest S-wave states with a single charmed quark.

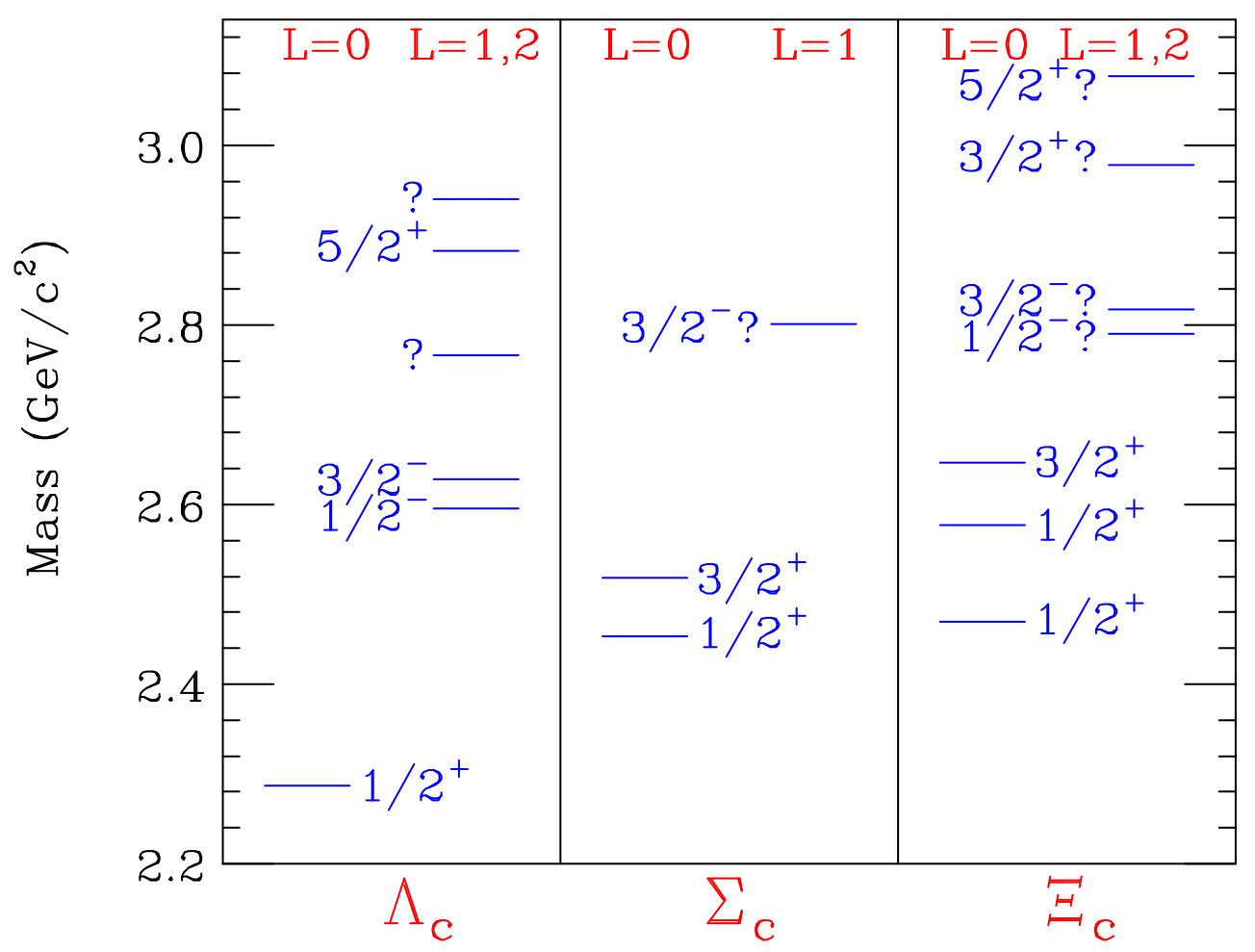

Figure 2: Singly-charmed baryons and some of their orbital excitations. 
$J^{P}=5 / 2^{+}[86]$, on the basis of the angular distribution in its $\Sigma_{c}(2455) \pi$ decays and the small measured ratio $\Gamma\left[\Sigma_{c}(2520) \pi\right] / \Gamma\left[\Sigma_{c}(2455) \pi\right] \simeq 1 / 4$.

(2) The highest $\Lambda_{c}$ was seen by BaBar in the decay mode $D^{0} p$ [85]. The Belle Collaboration has seen evidence for its decay to $\Sigma_{c}(2455) \pi$ [86].

(3) An excited $\Sigma_{c}$ candidate has been seen decaying to $\Lambda_{c} \pi^{+}$, with mass about $510 \mathrm{MeV}$ above $M\left(\Lambda_{c}\right)$ [87]. Its $J^{P}$ shown in Fig. 2 is a guess, using ideas of [39].

(4) The highest $\Xi_{c}$ levels were reported by the Belle Collaboration in Ref. [88], and confirmed by BaBar [89. Both are seen in the $\Lambda_{c}^{+} K^{-} \pi^{+}$channel. In the Belle data, a state with $M=2978.5 \pm 2.1 \pm 2.0 \mathrm{MeV} / c^{2}$ has width $\Gamma=43.5 \pm 7.5 \pm 7.0$ $\mathrm{MeV}$, while one with $M=3076.7 \pm 0.9 \pm 0.5 \mathrm{MeV} / c^{2}$ has width $\Gamma=6.2 \pm 1.2 \pm 0.8$ $\mathrm{MeV}$. The isospin partner of the higher state is also seen at $M=3082.8 \pm 1.8 \pm 1.5$ $\mathrm{MeV} / c^{2}$ in the $\Lambda_{c}^{+} K_{S} \pi^{-}$mode. The BaBar data are qualitatively similar, with the lower state having a width of $(23.6 \pm 2.8 \pm 1.3) \mathrm{MeV}$ and the higher having a width of $(6.2 \pm 1.6 \pm 0.5) \mathrm{MeV}$. The masses of these states suggest that they could have $L=2$, hence positive parity. If the light quarks in these states are coupled to spin 0 , as expected for the lowest orbital excitations, the allowed $J^{P}$ values are $3 / 2^{+}$and $5 / 2^{+}$. The disparity of the total widths suggests that the higher of the two may have $J^{P}=5 / 2^{+}$. Its decay to $\Sigma_{c}^{++} K^{-}$would then have to be via an $\mathrm{F}$-wave. (The $\Sigma_{c}^{*++} K^{-}$ channel, seen by BaBar [89], would be available to a P-wave.) If the lower state had $J^{P}=3 / 2^{+}$, it could decay to $\Sigma_{c}^{++} K^{-}$via a P-wave, and hence be broader.

\subsection{Excited charmed-strange states}

In the past couple of years the lowest $J^{P}=0^{+}$and $1^{+} c \bar{s}$ states turned out to have masses well below most expectations. If they had been as heavy as the alreadyseen $c \bar{s}$ states with $L=1$, the $D_{s 1}(2536)\left[J^{P}=1^{+}\right]$and $\left.D_{s 2}(2573)\left[J^{P}=2^{+}\right]\right)$, they would have been able to decay to $D \bar{K}$ (the $0^{+}$state) and $D^{*} \bar{K}$ (the $1^{+}$state). Instead several groups [90] observed a narrow $D_{s}(2317) \equiv D_{s 0}^{*}$ decaying to $\pi^{0} D_{s}$ and a narrow $D_{s}(2460) \equiv D_{s 1}^{*}$ decaying to $\pi^{0} D_{s}^{*}$, as illustrated in Fig. 3, Their low masses allow the isospin-violating and electromagnetic decays of $D_{s 0}^{*}$ and $D_{s 1}^{*}$ to be observable. The decays $D_{s}(2460) \rightarrow D_{s} \gamma$ and $D_{s}(2460) \rightarrow D_{s} \pi^{+} \pi^{-}$also have been seen [91, 92], and the absolute branching ratios $\mathcal{B}\left(D_{s 1}^{*} \rightarrow \pi^{0} D_{s}^{*}\right)=0.56 \pm 0.13 \pm 0.09$, $\mathcal{B}\left(D_{s 1}^{*} \rightarrow \gamma D_{s}\right)=0.16 \pm 0.04 \pm 0.03, \mathcal{B}\left(D_{s 1}^{*} \rightarrow \pi^{+} \pi^{-} D_{s}^{*}\right)=0.04 \pm 0.01$ measured.

The selection rules in decays of these states show that their $J^{P}$ values are consistent with $0^{+}$and $1^{+}$. Low masses are predicted [16] if these states are viewed as parity-doublets of the $D_{s}\left(0^{-}\right)$and $D_{s}^{*}\left(1^{-}\right) c \bar{s}$ ground states in the framework of chiral symmetry. The splitting from the ground states is $350 \mathrm{MeV}$ in each case. Alternatively, one can view these particles as bound states of $D^{(*)} K$, perhaps bound by the transitions $(c \bar{q})(q \bar{s}) \leftrightarrow(c \bar{s})$ (the binding energy in each case would be $41 \mathrm{MeV}$ ), or as $c \bar{s}$ states with masses lowered by coupling to $D^{(*)} K$ channels [93, 94].

A candidate for the first radial excitation of the $D_{s}^{*}(2112)$ has been observed in $B^{+} \rightarrow \bar{D}^{0} D^{0} K^{+}$decays 95 in the $M\left(D^{0} K^{+}\right)$spectrum. Its mass and width are $\left(2715 \pm 11_{-14}^{+11}\right)$ and $\left(115 \pm 20_{-32}^{+36}\right) \mathrm{MeV} / c^{2}$. Its spin-parity is $J^{P}=1^{-}$. It lies $\left(603_{-18}^{+16}\right)$ $\mathrm{MeV} / c^{2}$ above the ground state, in between the $2^{3} S_{1}-1^{3} S_{1}$ spacings of $(681 \pm 20)$ $\mathrm{MeV} / c^{2}$ for $s \bar{s}$ and $589 \mathrm{MeV} / c^{2}$ for $c \bar{c}$ [35]. This is as expected in an interpolating 


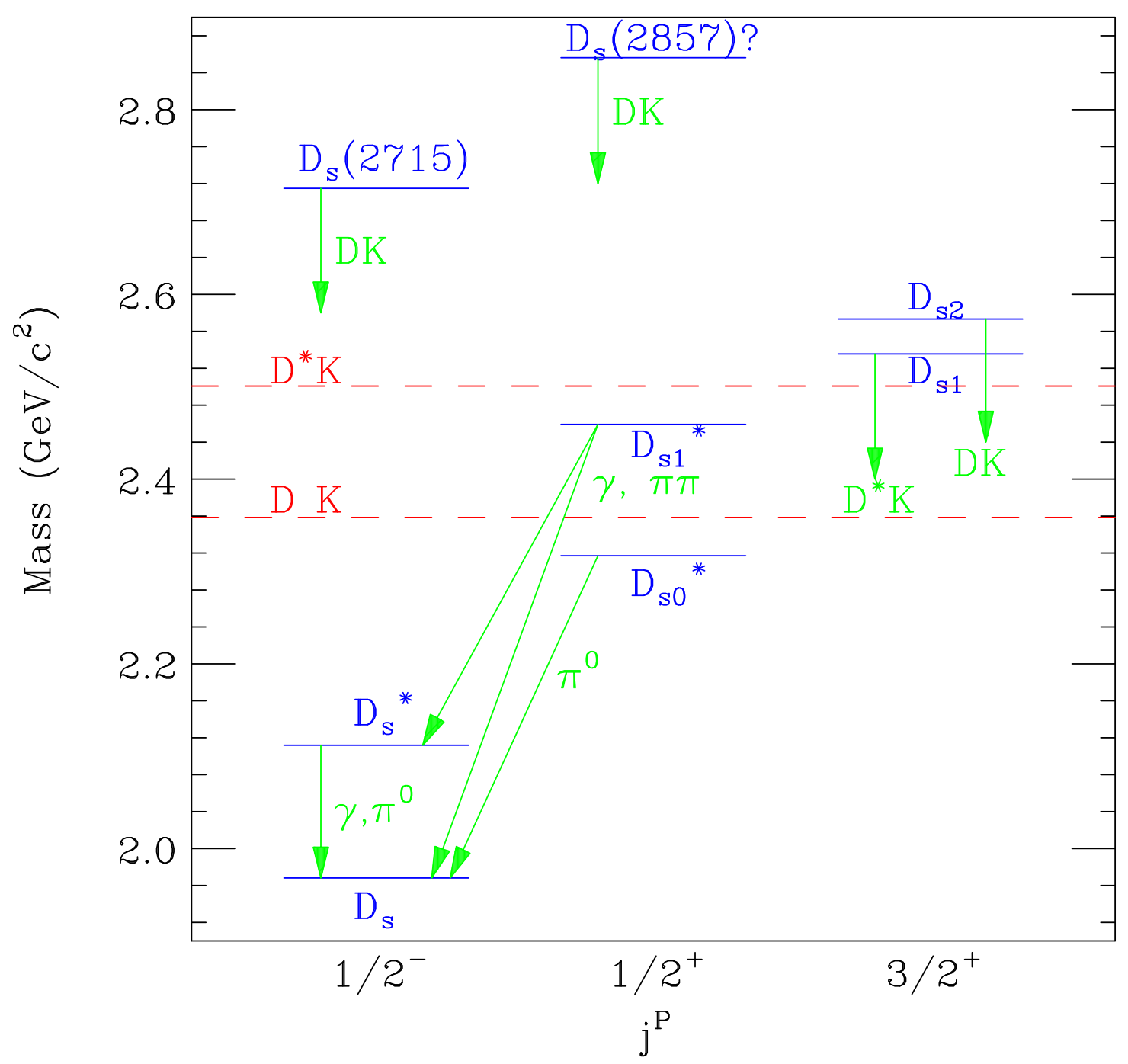

Figure 3: Charmed-strange mesons with $L=0$ (negative-parity), $L=1$ (positiveparity), and candidate for state with $L=2$ (positive parity). Here $j^{P}$ denotes the total light-quark spin + orbital angular momentum and the parity $P$.

potential such as a power-law with a small power [96], and as predicted in the quark model of Ref. [26]. (Ref. [97] prefers to identify this state as the lowest ${ }^{3} D_{1} c \bar{s}$ level on the basis of the ${ }^{3} P_{0}$ decay model mentioned earlier.)

An even higher-lying $c \bar{s}$ state has been observed by the BaBar Collaboration [98]. It is seen decaying to $D^{0} K^{+}$and $D^{+} K_{S}$, so it must have natural spin-parity $0^{+}$, $1^{-}, 2^{+}, \ldots$ Its mass and width are $(2856.6 \pm 1.5 \pm 5.0)$ and $(48 \pm 7 \pm 10) \mathrm{MeV} / \mathrm{c}^{2}$. It has been interpreted as a radial excitation of the $0^{+}$state $D_{s 0}(2317)$, i.e. the $0^{+}\left(2^{3} P_{0}\right)$ state [99, 100], shown hypothetically in Fig. 3, or a $3^{-}\left({ }^{3} D_{3}\right)$ state [101]. Both assignments are consistent with the ${ }^{3} P_{0}$ decay model [97]. 


\subsection{Excited charmed-nonstrange states}

In contrast to the lightest $0^{+}, 1^{+}$charmed-strange states, which are too light to decay to $D K$ or $D^{*} K$, the lightest $0^{+}, 1^{+}$charmed-nonstrange candidates appear to be heavy enough to decay to $D \pi$ or $D^{*} \pi$, and thus are expected to be broad. Heavy quark symmetry predicts the existence of a $0^{+}, 1^{+}$pair with light-quark total angular momentum and parity $j^{P}=1 / 2^{+}$decaying to $D \pi$ or $D^{*} \pi$, respectively, via an S-wave. A $1^{+}, 2^{+}$pair with $j^{P}=3 / 2^{+}$, decaying primarily via a $\mathrm{D}$-wave to $D^{*} \pi$ or both $D \pi$ and $D^{*} \pi$, respectively, has been well-represented for a number of years by states at $2422.3 \pm 1.3 \mathrm{MeV} / c^{2}$ and $2461.1 \pm 1.6 \mathrm{MeV} / c^{2}[35]$. The situation with regard to the $j^{P}=1 / 2^{+}$candidates is much less well-understood. CLEO [102 and Belle [103] find a broad $1^{+}$candidate in the range $2420-2460 \mathrm{MeV} / c^{2}$, while Belle and FOCUS [104] find broad $0^{+}$candidates near 2300 and $2400 \mathrm{MeV} / c^{2}$, respectively. For further details, see the review of Ref. [105].

\section{$4.4 \quad D^{+}$and $D_{s}$ decay constants}

CLEO has reported the first significant measurement of the $D^{+}$decay constant: $f_{D^{+}}=$ $\left(222.6 \pm 16.7_{-3.4}^{+2.8}\right) \mathrm{MeV}$ [106]. This is consistent with lattice predictions, including one [107] of $(201 \pm 3 \pm 17) \mathrm{MeV}$. The accuracy of the previous world average [35] $f_{D_{s}}=(267 \pm 33) \mathrm{MeV}$ has been improved by a BaBar value $f_{D_{s}}=283 \pm 17 \pm 7 \pm 14$ $\mathrm{MeV}\left[108\right.$ ] and a new CLEO value $f_{D_{s}}=280.1 \pm 11.6 \pm 6.0 \mathrm{MeV}$ [109]. The latter, when combined with CLEO's $f_{D}$, leads to $f_{D_{s}} / f_{D}=1.26 \pm 0.11 \pm 0.03$. A lattice prediction for $f_{D_{s}}$ [107] is $f_{D_{s}}=249 \pm 3 \pm 16 \mathrm{MeV}$, leading to $f_{D_{s}} / f_{D}=1.24 \pm 0.01 \pm 0.07$. One expects $f_{B_{s}} / f_{B} \simeq f_{D_{s}} / f_{D}$ so better measurements of $f_{D_{s}}$ and $f_{D}$ by CLEO will help validate lattice calculations and provide input for interpreting $B_{s}$ mixing. A desirable error on $f_{B_{s}} / f_{B} \simeq f_{D_{s}} / f_{D}$ is $\leq 5 \%$ for useful determination of CKM element ratio $\left|V_{t d} / V_{t s}\right|$, needing errors $\leq 10 \mathrm{MeV}$ on $f_{D_{s}}$ and $f_{D}$. The ratio $\left|V_{t d} / V_{t s}\right|=$ $0.2060 \pm 0.0007$ (exp) ${ }_{-0.0080}^{+0.0081}$ (theor) is implied by a recent CDF result on $B_{s}-\bar{B}_{s}$ mixing [110] combined with $B-\bar{B}$ mixing and $\xi \equiv\left(f_{B_{s}} \sqrt{B_{B_{s}}} / f_{B} \sqrt{B_{B}}\right)=1.21_{-0.035}^{+0.047}$ from the lattice [111. A simple quark model scaling argument anticipated $f_{D_{s}} / f_{D} \simeq f_{B_{s}} / f_{B} \simeq$

$\sqrt{m_{s} / m_{d}} \simeq 1.25$, where $m_{s} \simeq 485 \mathrm{MeV}$ and $m_{d} \simeq 310 \mathrm{MeV}$ are constituent quark masses 112 .

\section{BEAUTY/BOTTOM HADRONS}

It has become common to refer to the quantum number $B$ possessed by the bottom quark $b$ as "beauty." In accord with the convention whereby the strangeness $S$ of the $s$ quark is assigned to be $S(s)=-1$, we take $B(b)=-1, B(\bar{b})=+1$.

The spectrum of ground-state hadrons containing a single $b$ quark is shown in Fig. 4. The CDF Collaboration has published measurements of the $B_{s}$ and $\Lambda_{b}$ masses and the $B_{s}-B^{0}$ and $\Lambda_{b}-B^{0}$ mass differences which are of better precision than the current world averages [113], and now have nearly five times the data on which that publication was based. With the current data sample of $1 \mathrm{fb}^{-1} \mathrm{CDF}$ now has evidence for the long-sought $\Sigma_{b}$ and $\Sigma_{b}^{*}$ states [114] very near the masses predicted from the 


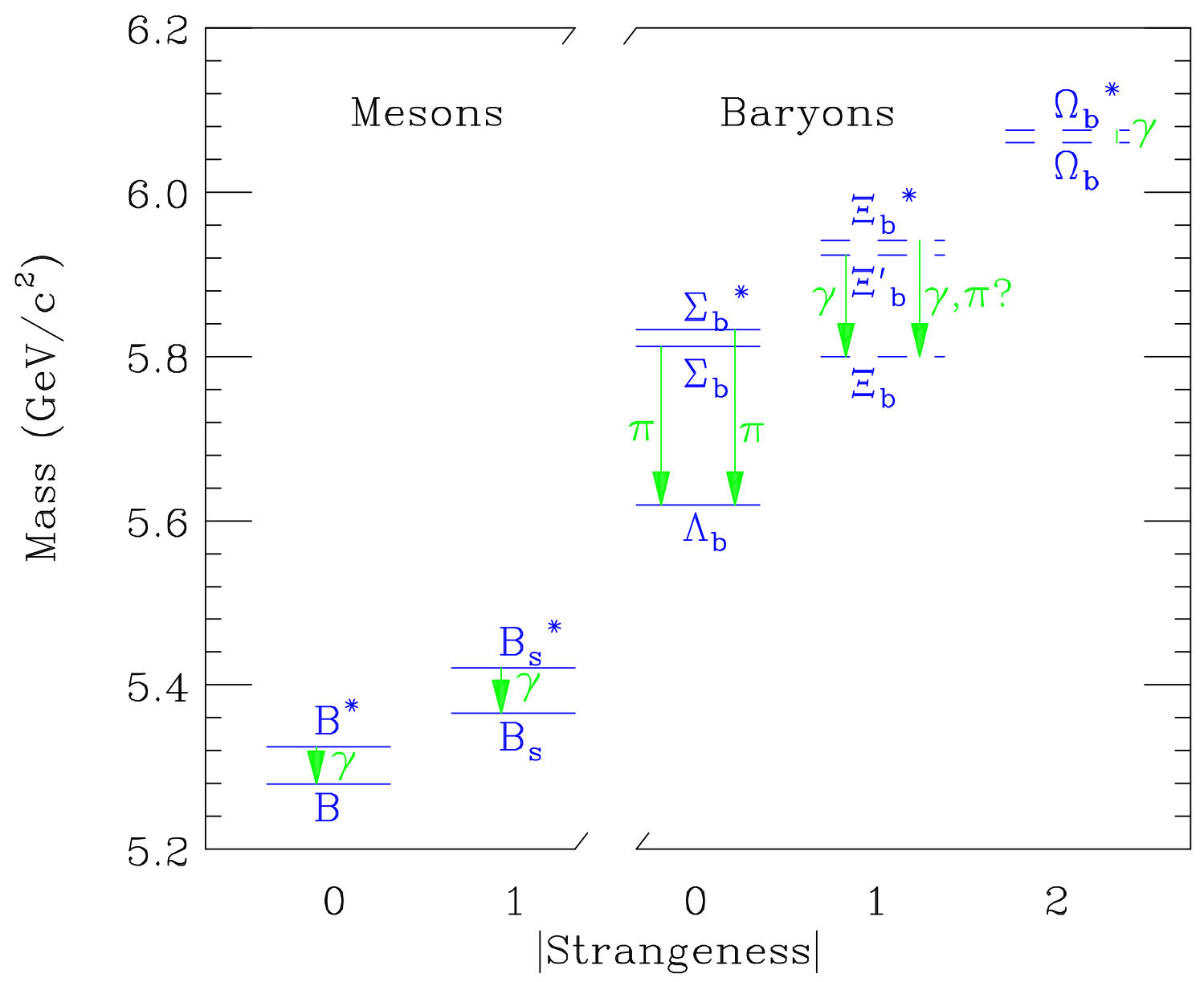

Figure 4: S-wave hadrons containing a single beauty quark. Dashed lines denote predicted levels not yet observed.

corresponding charmed baryons using heavy quark symmetry [115] or simple quarkmodel ideas [116]. Using measured mass differences and their value [113] of $M\left(\Lambda_{b}\right)=$ $5619.7 \pm 1.2 \pm 1.2 \mathrm{MeV} / c^{2}$, CDF reports

$$
\begin{array}{cll}
M\left(\Sigma_{b}^{-}\right) & =5815.2_{-0.9}^{+1.0} \pm 1.7 \mathrm{MeV} / c^{2}, & M\left(\Sigma_{b}^{+}\right)=5807.5_{-2.2}^{1.9} \pm 1.7 \mathrm{MeV} / c^{2} \\
M\left(\Sigma_{b}^{*-}\right) & =5836.7_{-2.3}^{+2.0} \pm 1.7 \mathrm{MeV} / c^{2}, & M\left(\Sigma_{b}^{*+}\right)=5829.0_{-1.7}^{+1.6} \pm 1.7 \mathrm{MeV} / c^{2}
\end{array}
$$

The analysis employs the assumption $M\left(\Sigma_{b}^{*-}\right)-M\left(\Sigma_{b}^{*+}\right)=M\left(\Sigma_{b}^{-}\right)-M\left(\Sigma_{b}^{+}\right)$, which was found in Ref. [117] to be valid to $0.4 \mathrm{MeV} / c^{2}$. The systematic error is dominated by uncertainty in $M\left(\Lambda_{b}\right)$.

The CDF Collaboration has identified events of the form $B_{c} \rightarrow J / \psi \pi^{ \pm}$, allowing a precise determination of the mass: $M=(6276.5 \pm 4.0 \pm 2.7) \mathrm{MeV} / c^{2}$ [118]. This is in reasonable accord with the latest lattice prediction of $6304 \pm 12_{-0}^{+18} \mathrm{MeV}$ [119].

The long-awaited $B_{s}-\bar{B}_{s}$ mixing has finally been observed [110, 120]. The CDF value, $\Delta m_{s}=17.77 \pm 0.10 \pm 0.07 \mathrm{ps}^{-1}$, constrains $f_{B_{s}}$ and $\left|V_{t d} / V_{t s}\right|$, as mentioned earlier.

The Belle Collaboration has observed the decay $B \rightarrow \tau \nu_{\tau}$ [121], leading to $f_{B}\left|V_{u b}\right|=$ $\left(10.1_{-1.4-1.3}^{+1.6+1.1}\right) \times 10^{-4} \mathrm{GeV}$. When combined with the value $\left|V_{u b}\right|=(4.39 \pm 0.33) \times 10^{-3}$ 
[122], this leads to $f_{B}=\left(229_{-31-34}^{+36+30}\right) \mathrm{MeV}$. A recent unquenched lattice estimate [123] is $f_{B}=(216 \pm 22) \mathrm{MeV}$.

A new CDF value for the $\Lambda_{b}$ lifetime, $\tau\left(\Lambda_{b}\right)=\left(1.593_{-0.078}^{+0.083} \pm 0.033\right) \mathrm{ps,}$, was reported recently [124]. Whereas the previous world average of $\tau\left(\Lambda_{b}\right)$ was about 0.8 that of $B^{0}$, below theoretical predictions, the new CDF value substantially increases the world average to a value $\tau\left(\Lambda_{b}\right)=(1.410 \pm 0.054)$ ps which is $0.923 \pm 0.036$ that of $B^{0}$ and quite comfortable with theory.

\section{CHARMONIUM}

\subsection{Observation of the $h_{c}$}

The $h_{c}\left(1^{1} P_{1}\right)$ state of charmonium has been observed by CLEO [125, 126] via $\psi(2 S) \rightarrow$ $\pi^{0} h_{c}$ with $h_{c} \rightarrow \gamma \eta_{c}$. Hyperfine splittings test the spin-dependence and spatial behavior of the $Q \bar{Q}$ force. Whereas these splittings are $M(J / \psi)-M\left(\eta_{c}\right) \simeq 115 \mathrm{MeV}$ for $1 \mathrm{~S}$ and $M\left[\psi^{\prime}\right]-M\left(\eta_{c}^{\prime}\right) \simeq 49 \mathrm{MeV}$ for $2 \mathrm{~S}$ levels, P-wave splittings should be less than a few $\mathrm{MeV}$ since the potential is proportional to $\delta^{3}(\vec{r})$ for a Coulomb-like $c \bar{c}$ interaction. Lattice QCD [127] and relativistic potential [29] calculations confirm this expectation. One expects $M\left(h_{c}\right) \equiv M\left(1^{1} P_{1}\right) \simeq\left\langle M\left({ }^{3} P_{J}\right)\right\rangle=3525.36 \pm 0.06 \mathrm{MeV}$.

Earlier $h_{c}$ sightings [125, 126] based on $\bar{p} p$ production in the direct channel, include a few events at $3525.4 \pm 0.8 \mathrm{MeV}$ seen in CERN ISR Experiment R704; a state at $3526.2 \pm 0.15 \pm 0.2 \mathrm{MeV}$, decaying to $\pi^{0} \mathrm{~J} / \psi$, reported by Fermilab E760 but not confirmed by Fermilab E835; and a state at $3525.8 \pm 0.2 \pm 0.2 \mathrm{MeV}$, decaying to $\gamma \eta_{c}$ with $\eta_{c} \rightarrow \gamma \gamma$, reported by E835 with about a dozen candidate events [128].

In the CLEO data, both inclusive and exclusive analyses see a signal near $\left\langle M\left({ }^{3} P_{J}\right)\right\rangle$. The exclusive analysis reconstructs $\eta_{c}$ in 7 decay modes, while no $\eta_{c}$ reconstruction is performed in the inclusive analysis. The exclusive signal is shown on the left in Fig. 5 , A total of 19 candidates were identified, with a signal of $17.5 \pm 4.5$ events above background. The mass and product branching ratio for the two transitions are $M\left(h_{c}\right)=$ $(3523.6 \pm 0.9 \pm 0.5) \mathrm{MeV} ; \mathcal{B}_{1}\left(\psi^{\prime} \rightarrow \pi^{0} h_{c}\right) \mathcal{B}_{2}\left(h_{c} \rightarrow \gamma \eta_{c}\right)=(5.3 \pm 1.5 \pm 1.0) \times 10^{-4}$ The result of one of two inclusive analyses is shown on the right in Fig. 5. These yield $M\left(h_{c}\right)=(3524.9 \pm 0.7 \pm 0.4) \mathrm{MeV}, \mathcal{B}_{1} \mathcal{B}_{2}=(3.5 \pm 1.0 \pm 0.7) \times 10^{-4}$. Combining exclusive and inclusive results yields $M\left(h_{c}\right)=(3524.4 \pm 0.6 \pm 0.4) \mathrm{MeV}$, $\mathcal{B}_{1} \mathcal{B}_{2}=(4.0 \pm 0.8 \pm 0.7) \times 10^{-4}$. The $h_{c}$ mass is $(1.0 \pm 0.6 \pm 0.4) \mathrm{MeV}$ below $\left\langle M\left({ }^{3} P_{J}\right)\right\rangle$, barely consistent with the (nonrelativistic) bound [129] $M\left(h_{c}\right) \geq\left\langle M\left({ }^{3} P_{J}\right)\right\rangle$ and indicating little $\mathrm{P}$-wave hyperfine splitting in charmonium. The value of $\mathcal{B}_{1} \mathcal{B}_{2}$ agrees with theoretical estimates of $\left(10^{-3} \cdot 0.4\right)$.

\subsection{Decays of the $\psi^{\prime \prime} \equiv \psi(3770)$}

The $\psi^{\prime \prime}(3770)$ is a potential "charm factory" for present and future $e^{+} e^{-}$experiments. At one time $\sigma\left(e^{+} e^{-} \rightarrow \psi^{\prime \prime}\right)$ seemed larger than $\sigma\left(e^{+} e^{-} \rightarrow \psi^{\prime \prime} \rightarrow D \bar{D}\right)$, raising the question of whether there were significant non- $D \bar{D}$ decays of the $\psi^{\prime \prime}$ [130]. A new CLEO measurement [131], $\sigma\left(\psi^{\prime \prime}\right)=\left(6.38 \pm 0.08_{-0.30}^{+0.41}\right) \mathrm{nb}$, appears very close to the

CLEO value $\sigma(D \bar{D})=\left(6.39 \pm 0.10_{-0.08}^{+0.17}\right)$ nb [132], leaving little room for non- $D \bar{D}$ 

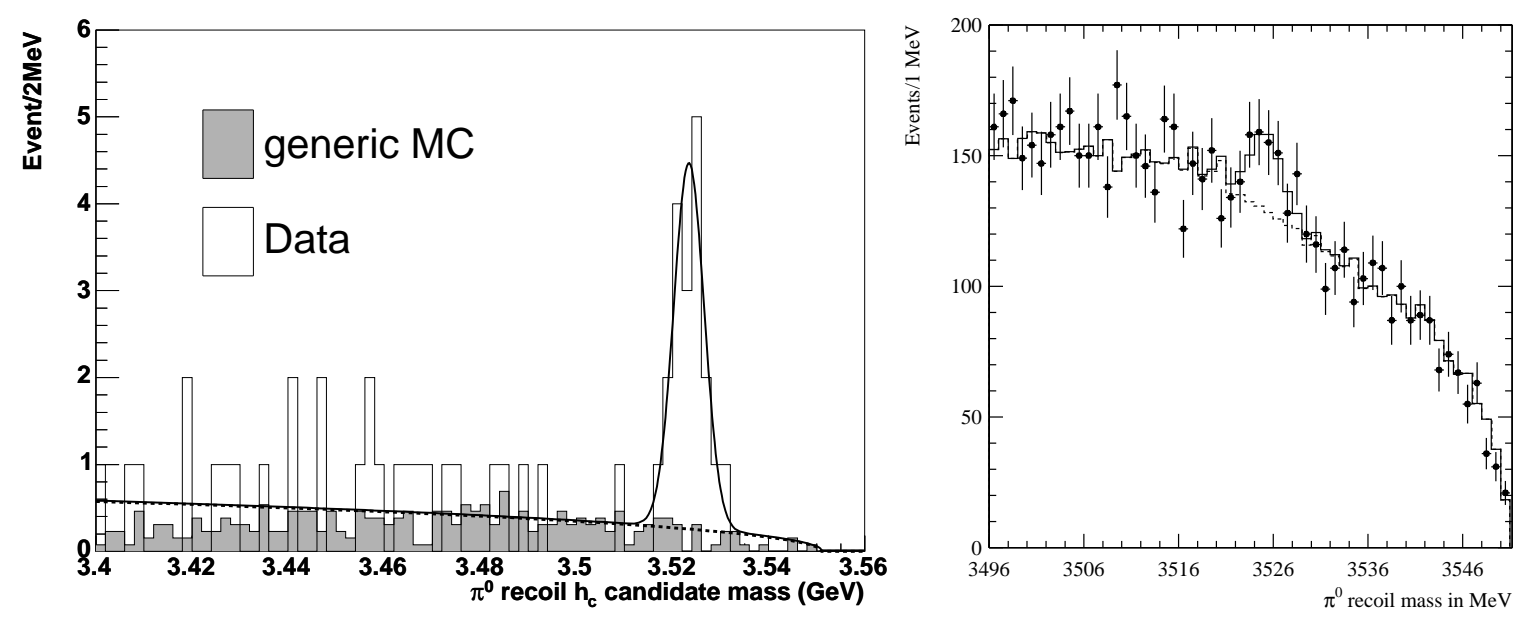

Figure 5: Left: Exclusive $h_{c}$ signal from CLEO (3 million $\psi(2 S)$ decays). Data events correspond to open histogram; Monte Carlo background estimate is denoted by shaded histogram. The signal shape is a double Gaussian, obtained from signal Monte Carlo. The background shape is an ARGUS function. Right: Inclusive $h_{c}$ signal from CLEO (3 million $\psi(2 S)$ decays). The curve denotes the background function based on generic Monte Carlo plus signal. The dashed line shows the contribution of background alone. Both figures are from Ref. [126].

Table I: CLEO results on radiative decays $\psi^{\prime \prime} \rightarrow \gamma \chi_{c J}$. Theoretical predictions of [31] are (a) without and (b) with coupled-channel effects; (c) shows predictions of [130].

\begin{tabular}{ccccc}
\hline Mode & \multicolumn{3}{c}{ Predicted (keV) } & CLEO \\
& (a) & (b) & (c) & {$[138]$} \\
\hline$\gamma \chi_{c 2}$ & 3.2 & 3.9 & $24 \pm 4$ & $<21$ \\
$\gamma \chi_{c 1}$ & 183 & 59 & $73 \pm 9$ & $75 \pm 18$ \\
$\gamma \chi_{c 0}$ & 254 & 225 & $523 \pm 12$ & $172 \pm 30$ \\
\hline
\end{tabular}

decays. (In recent BES analyses [133] a significant non- $D \bar{D}$ component could still be present.)

One finds that $\mathcal{B}\left(\psi^{\prime \prime} \rightarrow \pi \pi J / \psi, \gamma \chi_{c J}, \ldots\right)$ sum to at most $1-2 \%$. Moreover, both CLEO and BES [134], in searching for enhanced light-hadron modes, find only that the $\rho \pi$ mode, suppressed in $\psi(2 S)$ decays, also is suppressed in $\psi^{\prime \prime}$ decays.

Some branching ratios for $\psi^{\prime \prime} \rightarrow X J / \psi[135]$ are $\mathcal{B}\left(\psi^{\prime \prime} \rightarrow \pi^{+} \pi^{-} J / \psi\right)=(0.189 \pm$ $0.020 \pm 0.020) \%, \mathcal{B}\left(\psi^{\prime \prime} \rightarrow \pi^{0} \pi^{0} J / \psi\right)=(0.080 \pm 0.025 \pm 0.016) \%, \mathcal{B}\left(\psi^{\prime \prime} \rightarrow \eta J / \psi\right)=$ $(0.087 \pm 0.033 \pm 0.022) \%$, and $\mathcal{B}\left(\psi^{\prime \prime} \rightarrow \pi^{0} J / \psi\right)<0.028 \%$. The value of $\mathcal{B}\left[\psi^{\prime \prime}(3770) \rightarrow\right.$ $\pi^{+} \pi^{-} J / \psi$ ] found by CLEO is a bit above $1 / 2$ that reported by BES [136]. These account for less than $1 / 2 \%$ of the total $\psi^{\prime \prime}$ decays.

CLEO has reported results on $\psi^{\prime \prime} \rightarrow \gamma \chi_{c J}$ partial widths, based on the exclusive process $\psi^{\prime \prime} \rightarrow \gamma \chi_{c 1,2} \rightarrow \gamma \gamma J / \psi \rightarrow \gamma \gamma \ell^{+} \ell^{-}$[137] and reconstruction of exclusive $\chi_{c J}$ decays [138]. The results are shown in Table \. implying $\sum_{J} \mathcal{B}\left(\psi^{\prime \prime} \rightarrow \gamma \chi_{c J}\right)=\mathcal{O}(1 \%)$. 
Several searches for $\psi^{\prime \prime}(3770) \rightarrow$ (light hadrons), including VP, $K_{L} K_{S}$, and multibody final states have been performed. Two CLEO analyses [139, 140] find no evidence for any light-hadron $\psi^{\prime \prime}$ mode above expectations from continuum production except $\phi \eta$, indicating no obvious signature of non- $D \bar{D} \psi^{\prime \prime}$ decays.

\section{3 $X(3872):$ A $1^{++}$molecule}

Many charmonium states above $D \bar{D}$ threshold have been seen recently. Reviews may be found in Refs. [141, 142]. The $X(3872)$, discovered by Belle in $B$ decays [143] and confirmed by BaBar [144] and in hadronic production [145], was seen first in its decay to $J / \psi \pi^{+} \pi^{-}$. Since it lies well above $D \bar{D}$ threshold but is narrower than experimental resolution (a few $\mathrm{MeV}$ ), unnatural $J^{P}=0^{-}, 1^{+}, 2^{-}$is favored. It has many features in common with an S-wave bound state of $\left(D^{0} \bar{D}^{* 0}+\bar{D}^{0} D^{* 0}\right) / \sqrt{2} \sim c \bar{c} u \bar{u}$ with $J^{P C}=1^{++}$[146]. The simultaneous decay of $X(3872)$ to $\rho J / \psi$ and $\omega J / \psi$ with roughly equal branching ratios is a consequence of this "molecular" assignment.

Analysis of angular distributions [147] in $X \rightarrow \rho J / \psi, \omega J / \psi$ favors the $1^{++}$assignment [148]. (See also [91, 142].) Although a high-statistics analysis by CDF cannot distinguish between $J^{P C}=1^{++}$or $2^{-+}$[149], the latter assignment is disfavored by Belle's observation [150] of $X \rightarrow D^{0} \bar{D}^{0} \pi^{0}$, which would require at least two units of relative orbital angular momentum in the three-body state, very near threshold.

The detection of the $\gamma J / \psi$ mode $\left(\sim 14 \%\right.$ of $\left.J / \psi \pi^{+} \pi^{-}\right)$[151] confirms the assignment of positive $C$ and suggests a $c \bar{c}$ admixture in the wave function. BaBar [152] finds $\mathcal{B}\left[X(3872) \rightarrow \pi^{+} \pi^{-} J / \psi\right]>0.042$ at $90 \%$ c.l.

\subsection{Additional states around $3940 \mathrm{MeV}$}

Belle has reported a candidate for a $2^{3} P_{2}\left(\chi_{c 2}^{\prime}\right)$ state in $\gamma \gamma$ collisions [153], decaying to $D \bar{D}$. The angular distribution of $D \bar{D}$ pairs is consistent with $\sin ^{4} \theta^{*}$ as expected for a state with $J=2, \lambda= \pm 2$. It has $M=3929 \pm 5 \pm 2 \mathrm{MeV}, \Gamma=29 \pm 10 \pm 3 \mathrm{MeV}$, and $\Gamma_{e e} \mathcal{B}(D \bar{D})=0.18 \pm 0.06 \pm 0.03 \mathrm{eV}$, all reasonable for a $\chi_{c 2}^{\prime}$ state.

A charmonium state $X(3938)$ is produced recoiling against $J / \psi$ in $e^{+} e^{-} \rightarrow J / \psi+$ $X$ 154 and is seen decaying to $D \bar{D}^{*}+$ c.c. Since all lower-mass states observed in this recoil process have $J=0$ (these are the $\eta_{c}(1 S), \chi_{c 0}$ and $\eta_{c}^{\prime}(2 S)$ ), it is tempting to identify this state with $\eta_{c}(3 S)$ (not $\chi_{c 0}^{\prime}$, which would decay to $D \bar{D}$ ).

The $\omega J / \psi$ final state in $B \rightarrow K \omega J / \psi$ shows a peak above threshold at $M(\omega J / \psi) \simeq$ $3940 \mathrm{MeV}$ [155]. This could be a candidate for one or more excited P-wave charmonium states, likely the $\chi_{c 1,2}^{\prime}\left(2^{3} P_{1,2}\right)$. The corresponding $b \bar{b}$ states $\chi_{b 1,2}^{\prime}$ have been seen to decay to $\omega \Upsilon(1 S)[156]$.

\subsection{The $Y(4260)$}

Last year BaBar reported a state $Y(4260)$ produced in the radiative return reaction $e^{+} e^{-} \rightarrow \gamma \pi^{+} \pi^{-} J / \psi$ and seen in the $\pi^{+} \pi^{-} J / \psi$ spectrum [157]. Its mass is consistent with being a $4 S$ level [158] since it lies about $230 \mathrm{MeV}$ above the $3 S$ candidate (to be compared with a similar $4 S-3 S$ spacing in the $\Upsilon$ system). Indeed, a $4 S$ charmonium level at $4260 \mathrm{MeV} / \mathrm{c}^{2}$ was anticipated on this basis [22]. With this assignment, the 

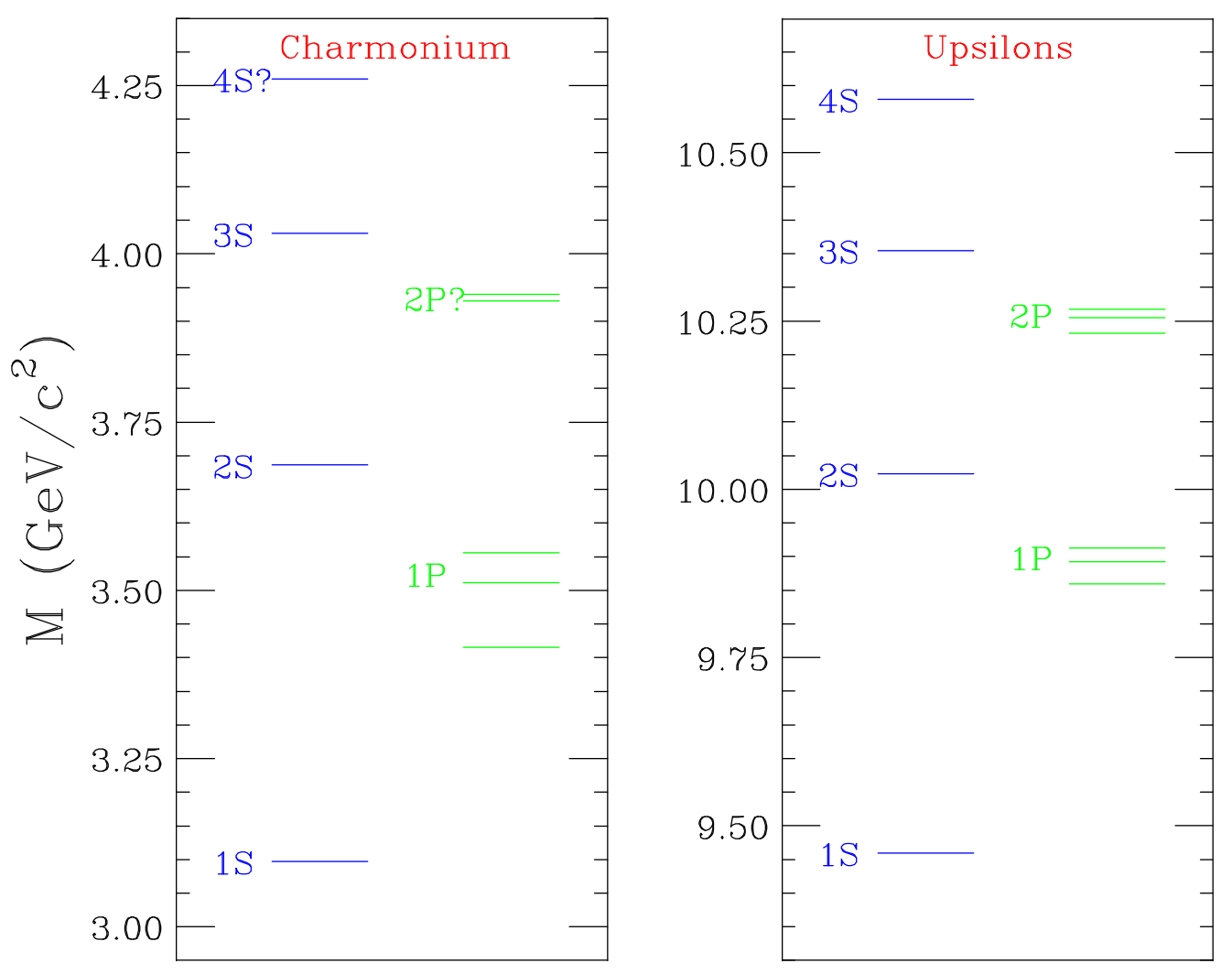

Figure 6: Congruence of charmonium and bottomonium spectra if the $Y(4260)$ is a $4 \mathrm{~S}$ level.

$n S$ levels of charmonium and bottomonium are nearly congruent to one another, as shown in Fig. 6. Their spacings would be identical if the interquark potential were $V(r) \sim \log (r)$, which may be viewed as an interpolation between the shortdistance $\sim-1 / r$ and long-distance $\sim r$ behavior expected in QCD. Circumstances weighing against the $4 S$ assignment include the lack of a peak in $R \equiv \sigma\left(e^{+} e^{-} \rightarrow\right.$ hadrons) $/ \sigma\left(e^{+} e^{-} \rightarrow \mu^{+} \mu^{-}\right)$at $4260 \mathrm{MeV}$ ( $R$ exhibits a dip just below this energy), and a $4 S$ candidate at $4415 \mathrm{MeV} / c^{2}$ in accord with many potential models.

The CLEO Collaboration has confirmed the $Y(4260)$, both in a direct scan [161] and in radiative return [162]. Signals are seen for $Y(4260) \rightarrow \pi^{+} \pi^{-} J / \psi(11 \sigma)$, $\pi^{0} \pi^{0} J / \psi(5.1 \sigma)$, and $K^{+} K^{-} J / \psi(3.7 \sigma)$. There are also weak signals for $\psi(4160) \rightarrow$ $\pi^{+} \pi^{-} J / \psi(3.6 \sigma)$ and $\pi^{0} \pi^{0} J / \psi(2.6 \sigma)$, consistent with the $Y(4260)$ tail, and for $\psi(4040) \rightarrow \pi^{+} \pi^{-} J / \psi(3.3 \sigma)$.

Other interpretations of $Y(4260)$ include a $c s \bar{c} \bar{s}$ state [159] and a hybrid $c \bar{c} g$ state [160], for which it lies in the expected mass range. One consequence of the hybrid interpretation is a predicted decay to $D \bar{D}_{1}+$ c.c., where $D_{1}$ is a $\mathrm{P}$-wave $c \bar{q}$ pair. Now, $D \bar{D}_{1}$ threshold is $4287 \mathrm{MeV} / c^{2}$ if we consider the lightest $D_{1}$ to be the state noted in Ref. [35] at $2422 \mathrm{MeV} / c^{2}$. In this case the $Y(4260)$ would be a $D \bar{D}_{1}+$ c.c. bound state. It would decay to $D \pi \bar{D}^{*}$, where the $D$ and $\pi$ are not in a $D^{*}$. The dip in $R_{e^{+} e^{-}}$ lies just below $D \pi \bar{D}^{*}$ threshold, which may be the first S-wave meson pair accessible in $c \bar{c}$ fragmentation [163]. 


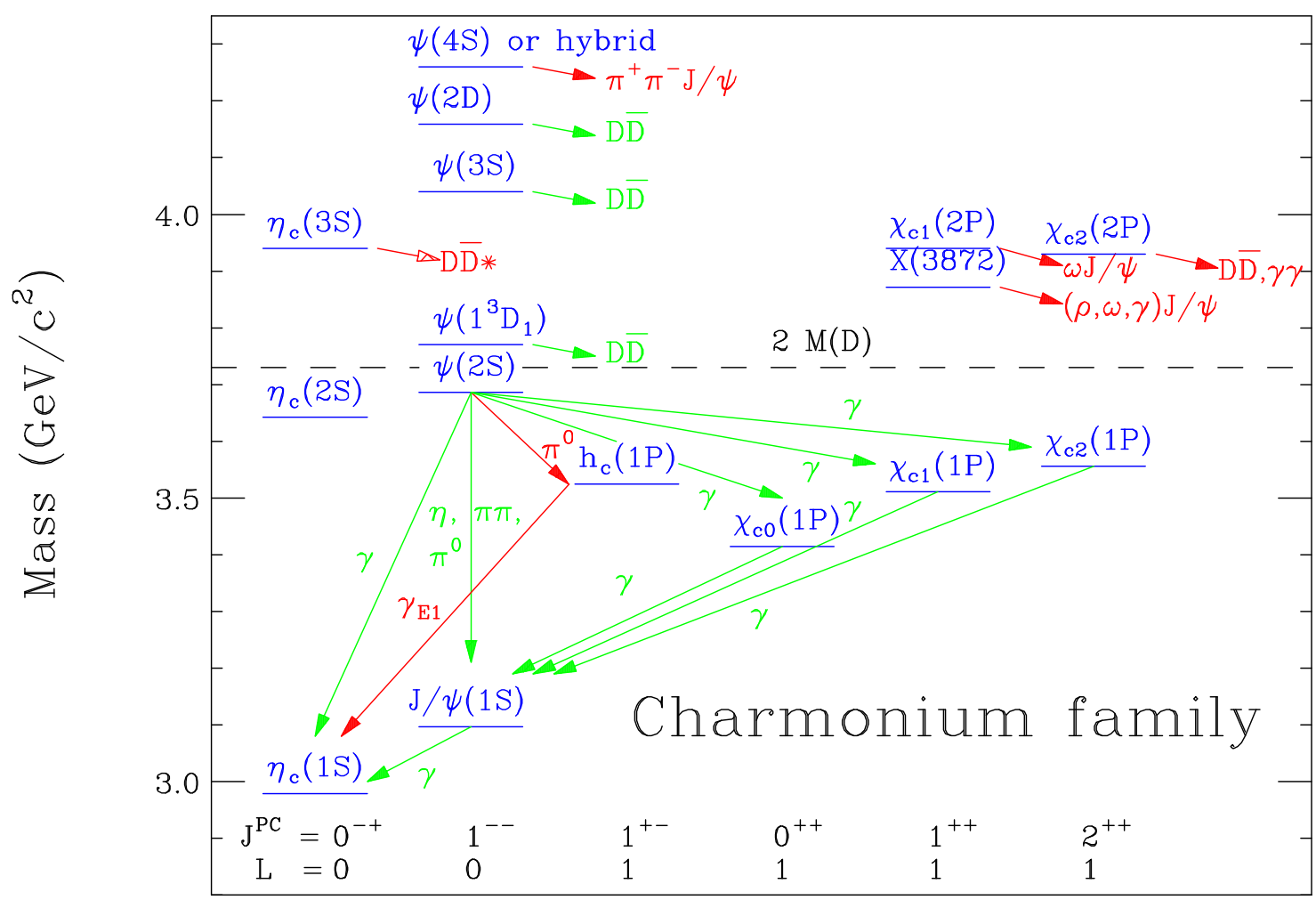

Figure 7: Charmonium states including levels above charm threshold.

\subsection{Charmonium: updated}

Remarkable progress has been made in the spectroscopy of charmonium states above charm threshold in the past few years. Fig. 7 summarizes the levels (some of whose assignments are tentative). Even though such states can decay to charmed pairs (with the possible exception of $X(3872)$, which may be just below $D \bar{D}^{*}$ threshold), other decay modes are being seen.

\section{THE $\Upsilon$ FAMILY (BOTTOMONIUM)}

Some properties and decays of the $\Upsilon(b \bar{b})$ levels are summarized in Fig. 8, Masses are in agreement with unquenched lattice QCD calculations [164]. Direct photons have been observed in $1 \mathrm{~S}, 2 \mathrm{~S}$, and $3 \mathrm{~S}$ decays, implying estimates of the strong fine-structure constant consistent with others [165]. The transitions $\chi_{b}(2 P) \rightarrow \pi \pi \chi_{b}(1 P)$ have been seen [166, 167]. BaBar has measured the partial widths $\Gamma\left[\Upsilon(4 S) \rightarrow \pi^{+} \pi^{-} \Upsilon(1 S)\right]=$ $1.8 \pm 0.4 \mathrm{keV}$ and $\Gamma\left[\Upsilon(4 S) \rightarrow \pi^{+} \pi^{-} \Upsilon(2 S)\right]=2.7 \pm 0.8 \mathrm{keV}$ [168], while Belle has seen $\Upsilon(4 S) \rightarrow \pi^{+} \pi^{-} \Upsilon(1 S)$, with a branching ratio $\mathcal{B}=(1.1 \pm 0.2 \pm 0.4) \times 10^{-4}$ [169].

\subsection{Remeasurement of $\Upsilon(n S)$ properties}

New values of $\mathcal{B}\left[\Upsilon(1 S, 2 S, 3 S) \rightarrow \mu^{+} \mu^{-}\right]=(2.49 \pm 0.02 \pm 0.07,2.03 \pm 0.03 \pm 0.08,2.39 \pm$ $0.07 \pm 0.10) \%$ [170], when combined with new measurements $\Gamma_{e e}(1 S, 2 S, 3 S)=(1.252 \pm$ 


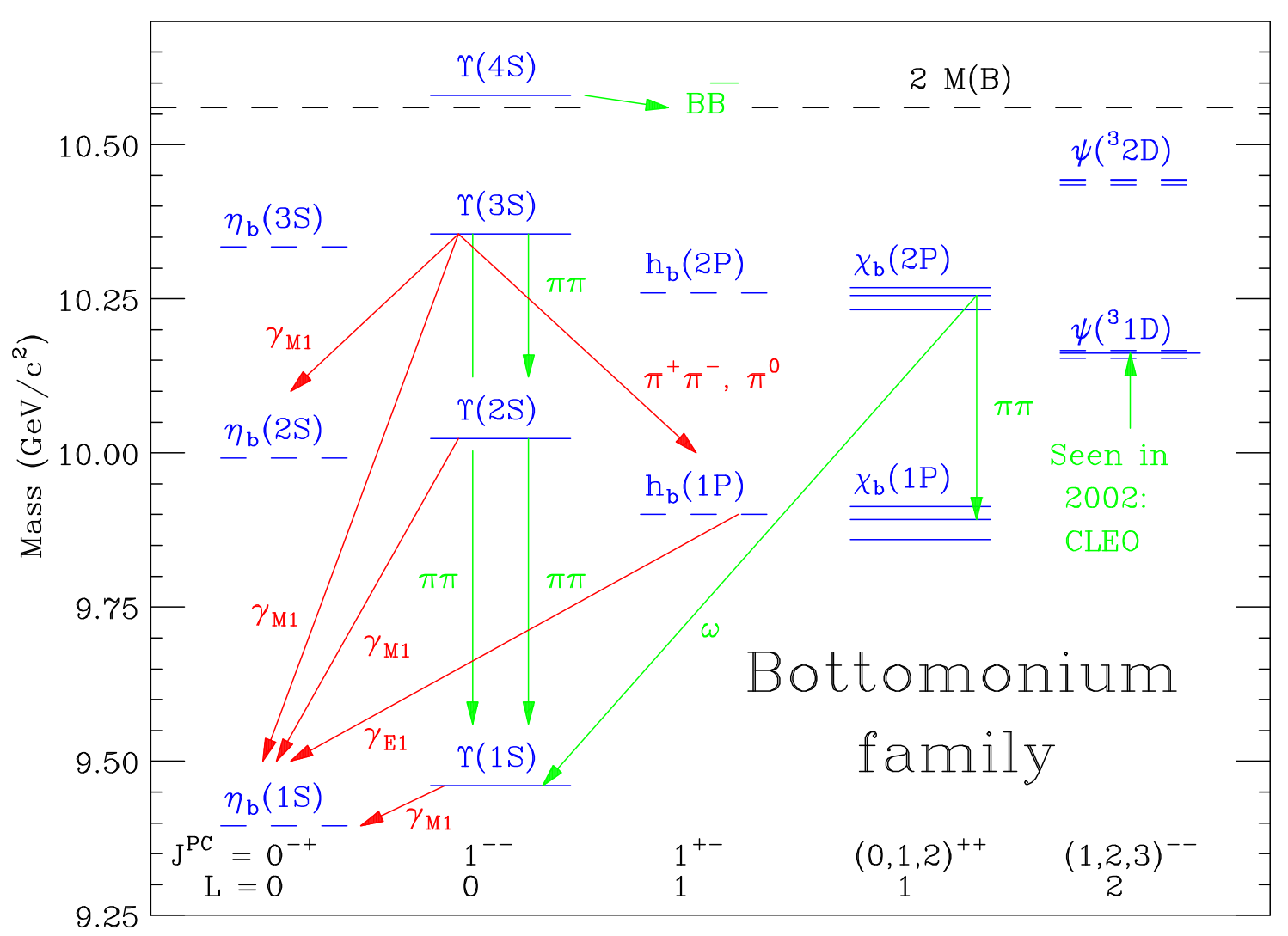

Figure 8: Bottomonium levels and some decays. Electric dipole (E1) transitions $S \leftrightarrow P \leftrightarrow D$ are not shown.

$0.004 \pm 0.019,0.581 \pm 0.004, \pm 0.009,0.413 \pm 0.004 \pm 0.006) \mathrm{keV}$ imply total widths $\Gamma_{\text {tot }}(1 S, 2 S, 3 S)=(50.3 \pm 1.7,28.6 \pm 1.3,17.3 \pm 0.6) \mathrm{keV}$. The values of $\Gamma_{\text {tot }}(2 S, 3 S)$ changed considerably with respect to previous world averages. Combining with previous data, the Particle Data Group [35] now quotes $\Gamma_{\text {tot }}(1 S, 2 S, 3 S)=(54.02 \pm$ $1.25,31.98 \pm 2.63,20.32 \pm 1.85) \mathrm{keV}$, which we shall use in what follows. This will lead to changes in comparisons of predicted and observed transition rates. As one example, the study of $\Upsilon(2 S, 3 S) \rightarrow \gamma X$ decays [171] has provided new branching ratios for $\mathrm{E} 1$ transitions to $\chi_{b J}(1 P), \chi_{b J}^{\prime}(2 P)$ states. These may be combined with the new total widths to obtain updated partial decay widths [line (a) in Table II], which may be compared with one set of non-relativistic predictions [172] [line (b)]. The suppression of transitions to $J=0$ states by $10-20 \%$ with respect to nonrelativistic expectations agrees with relativistic predictions [173]. The partial width for $\Upsilon(3 S) \rightarrow \gamma 1^{3} P_{0}$ is found to be $61 \pm 23 \mathrm{eV}$, about nine times the highly-suppressed value predicted in Ref. [172]. That prediction is very sensitive to details of wave functions; the discrepancy indicates the importance of relativistic distortions.

\section{2 $\quad b \bar{b}$ spin singlets}

Decays of the $\Upsilon(1 S, 2 S, 3 S)$ states are potential sources of information on $b \bar{b}$ spinsinglets, but none has been seen yet. One expects $1 \mathrm{~S}, 2 \mathrm{~S}$, and $3 \mathrm{~S}$ hyperfine splittings 
Table II: Comparison of observed (a) and predicted (b) partial widths for $2 S \rightarrow 1 P_{J}$ and $3 S \rightarrow 2 P_{J}$ transitions in $b \bar{b}$ systems.

\begin{tabular}{|c|ccc|ccc|}
\hline & \multicolumn{2}{|c|}{$\Gamma(\mathrm{keV}), 2 S \rightarrow 1 P_{J}$ transitions } & \multicolumn{3}{c|}{$\Gamma(\mathrm{keV}), 3 S \rightarrow 2 P_{J}$ transitions } \\
& $J=0$ & $J=1$ & $J=2$ & $J=0$ & $J=1$ & $J=2$ \\
\hline (a) & $1.20 \pm 0.18$ & $2.22 \pm 0.23$ & $2.32 \pm 0.23$ & $1.38 \pm 0.19$ & $2.95 \pm 0.30$ & $3.21 \pm 0.33$ \\
(b) & 1.39 & 2.18 & 2.14 & 1.65 & 2.52 & 2.78 \\
\hline \hline
\end{tabular}

to be approximately $60,30,20 \mathrm{MeV} / c^{2}$, respectively [174]. The lowest P-wave singlet state (" $h_{b}$ ") is expected to be near $\left\langle M\left(1^{3} P_{J}\right)\right\rangle \simeq 9900 \mathrm{MeV} / c^{2}$ [175].

Several searches have been performed or are under way in $1 \mathrm{~S}, 2 \mathrm{~S}$, and $3 \mathrm{~S}$ CLEO data. One can search for the allowed M1 transition in $\Upsilon(1 S) \rightarrow \gamma \eta_{b}(1 S)$ by reconstructing exclusive final states in $\eta_{b}(1 S)$ decays and dispensing with the soft photon, which is likely to be swallowed up in background. Final states are likely to be of high multiplicity.

One can search for higher-energy but suppressed M1 photons in $\Upsilon\left(n^{\prime} S\right) \rightarrow \gamma \eta_{b}(n S)$ $\left(n \neq n^{\prime}\right)$ decays. These searches already exclude many models. The strongest upper limit obtained is for $n^{\prime}=3, n=1: \mathcal{B} \leq 4.3 \times 10^{-4}$ (90\% c.l.). $\eta_{b}$ searches using sequential processes $\Upsilon(3 S) \rightarrow \pi^{0} h_{b}\left(1^{1} P_{1}\right) \rightarrow \pi^{0} \gamma \eta_{b}(1 S)$ and $\Upsilon(3 S) \rightarrow \gamma \chi_{b 0}^{\prime} \rightarrow \gamma \eta \eta_{b}(1 S)$ (the latter suggested in Ref. [176]) are being conducted but there are no results yet. Additional searches for $h_{b}$ involve the transition $\Upsilon(3 S) \rightarrow \pi^{+} \pi^{-} h_{b}$ [for which a typical experimental upper bound based on earlier CLEO data [177] is $\mathcal{O}\left(10^{-3}\right)$ ], with a possible $h_{b} \rightarrow \gamma \eta_{b}$ transition expected to have a $40 \%$ branching ratio [175].

\section{FUTURE PROSPECTS}

Two main sources of information on hadron spectroscopy in the past few years have been BES-II and CLEO. BES-II has ceased operation to make way for BES-III. CLEO plans to focus on center-of-mass energies of 3770 and $4170 \mathrm{MeV}$, split roughly equally, with goals of about $750 \mathrm{pb}^{-1}$ at each energy. The determination of $f_{D}, f_{D_{s}}$, and form factors for semileptonic $D$ and $D_{s}$ decays will provide incisive tests for lattice gauge theories and measure CKM factors $V_{c d}$ and $V_{c s}$ with unprecedented precision. In addition, about 25 million $\psi(2 S)$ (about 8 times the current CLEO sample) have been accumulated in the summer of 2006. CLEO-c running will end at the end of March 2008; BES-III and PANDA will extend charm studies further.

Belle has taken $3 \mathrm{fb}^{-1}$ of data at $\Upsilon(3 S)$ [CLEO has $(1.1,1.2,1.2) \mathrm{fb}^{-1}$ at $(1 \mathrm{~S}, 2 \mathrm{~S}, 3 \mathrm{~S})$ ]. Both BaBar and Belle are well-situated to study hadron spectroscopy. We look forward to further contributions from CDF and D0, and to the LHCb experiment, with its detailed studies of charm and $b$ hadrons produced in hadronic collisions. 


\section{SUMMARY}

Hadron spectroscopy is providing both long-awaited states like $h_{c}$ and surprises like low-lying P-wave $D_{s}$ mesons, X(3872), X(3940), Y(3940), Z(3940) and Y(4260). Decays of $\psi^{\prime \prime}(3770)$ shed light on its nature. Higher excitations of charmed baryons and charmed-strange mesons continue to be uncovered. Upon reflection, some properties of the new hadron states may be less surprising but we are continuing to learn about properties of QCD in the strong-coupling regime. There is evidence for molecules, 3S, $2 \mathrm{P}, 4 \mathrm{~S}$ or hybrid charmonium, and interesting decays of states above flavor threshold.

QCD may not be the last strongly coupled theory with which we have to deal. The mystery of electroweak symmetry breaking may require related techniques, if the long-awaited Higgs boson is not elementary but a phenomenon emerging from a new strongly-interacting sector (as in the "technicolor" scheme or other pictures of composite Higgs bosons). Understanding the very structure of quarks and leptons may require spectroscopic techniques. This will be a very different spectroscopy than that of hadrons, since all evidence points to a large separation between the masses of the known elementary fermions and any possible compositeness scale. The insights on hadron spectra described here are coming to us in general from experiments at the frontier of intensity and detector capabilities rather than energy, illustrating the importance of a diverse approach to the fundamental structure of matter.

\section{ACKNOWLEDGMENTS}

I thank colleagues on BaBar, Belle, and CLEO for sharing data and for helpful discussions, and E. van Beveren, D. Bugg, F. Close, L. Glozman, J. Hedditch, J. Peláez, and G. Rupp for some helpful references. This work was supported in part by the United States Department of Energy under Grant No. DE FG02 90ER40560.

\section{References}

[1] D. J. Gross and F. Wilczek, Phys. Rev. Lett. 30, 1343 (1973); Phys. Rev. D 8, 3633 (1973); H. D. Politzer, Phys. Rev. Lett. 30, 1346 (1973); Phys. Rept. 14, 129 (1974).

[2] E. D. Bloom et al., Phys. Rev. Lett. 23, 930 (1969); M. Breidenbach et al., Phys. Rev. Lett. 23, 935 (1969).

[3] J. D. Bjorken, Phys. Rev. 148, 1467 (1966); 160, 1582(E) (1967); Phys. Rev. Lett. 16, 408 (1966); Phys. Rev. 179, 1547 (1969); J. D. Bjorken and E. A. Paschos, Phys. Rev. 185, 1975 (1969);

[4] H. Lee and V. Burkert, this issue.

[5] E. Eichten, S. Godfrey, H. Mahlke and J. L. Rosner, "Quarkonia and their transitions," hep-ph/0701208. 
[6] S. Bethke, hep-ex/0606035; S. Kluth, hep-ex/0609020, presented at ICHEP 06 (Moscow, Russia, 2006), Proceedings edited by A. N. Sissakian and G. A. Kozlov, to be published by World Scientific, 2007; M. Davier, A. Höcker and Z. Zhang, hep-ph/0701170.

[7] C. T. H. Davies et al. [HPQCD Collaboration], Phys. Rev. Lett. 92, 022001 (2004).

[8] S. Weinberg, Phys. Rev. Lett. 17, 616 (1966).

[9] L. S. Brown and R. L. Goble, Phys. Rev. D 4, 723 (1971).

[10] S. M. Roy, Phys. Lett. 36B, 353 (1971).

[11] J. Gasser and H. Leutwyler, Annals Phys. 158, 142 (1984); Nucl. Phys. B 250, 465 (1985).

[12] V. Bernard and U. G. Meißner, Ann. Rev. Nucl. Part. Sci., manuscript in preparation.

[13] L. Ya. Glozman, Phys. Lett. B 475, 329 (2000); Int. J. Mod. Phys. A 21, 475 (2000); hep-ph/0701081; T. D. Cohen and L. Ya. Glozman, Int. J. Mod. Phys. A 17, 1327 (2002); L. Ya. Glozman and A. V. Nefdiev, Phys. Rev. D 73, 074018 (2006); R. L. Jaffe, D. Pirjol and A. Scardicchio, Phys. Rev. Lett. 96, 121601 (2006); Phys. Rept. 435, 157 (2006); Phys. Rev. D 74, 057901 (2006).

[14] S. Nussinov and W. Wetzel, Phys. Rev. D 36, 130 (1987).

[15] N. Isgur and M. B. Wise, Phys. Lett. B 232, 113 (1989); Phys. Lett. B 237, 527 (1990); Phys. Rev. D 42, 2388 (1990); Phys. Rev. Lett. 66, 1130 (1991).

[16] M. A. Nowak, M. Rho and I. Zahed, Phys. Rev. D 48, 4370 (1993); W. A. Bardeen and C. T. Hill, Phys. Rev. D 49, 409 (1994); W. A. Bardeen, E. J. Eichten and C. T. Hill, Phys. Rev. D 68, 054024 (2003).

[17] E. Eichten, K. Gottfried, T. Kinoshita, J. B. Kogut, K. D. Lane and T. M. Yan, Phys. Rev. Lett. 34, 369 (1975) [Erratum-ibid. 36, 1276 (1976)]; E. Eichten, K. Gottfried, T. Kinoshita, K. D. Lane and T. M. Yan, Phys. Rev. Lett. 36, 500 (1976); E. Eichten, K. Gottfried, T. Kinoshita, K. D. Lane and T. M. Yan, Phys. Rev. D 17, 3090 (1978) [Erratum-ibid. D 21, 313 (1980)]; Phys. Rev. D 21, 203 (1980).

[18] V. A. Novikov, L. B. Okun, M. A. Shifman, A. I. Vainshtein, M. B. Voloshin and V. I. Zakharov, Phys. Rept. 41, 1 (1978).

[19] T. Appelquist, R. M. Barnett and K. D. Lane, Ann. Rev. Nucl. Part. Sci. 28, 387 (1978).

[20] C. Quigg and J. L. Rosner, Phys. Rept. 56, 167 (1979). 
[21] H. Grosse and A. Martin, Phys. Rept. 60, 341 (1980).

[22] C. Quigg and J. L. Rosner, Phys. Lett. B 71, 153 (1977).

[23] R. Van Royen and V. F. Weisskopf, Nuovo Cim. A 50, 617 (1967) [Erratum-ibid. A 51, 583 (1967)].

[24] W. Kwong, C. Quigg, and J. L. Rosner, Ann. Rev. Nucl. Part. Sci. 37, 325 (1987).

[25] N. Brambilla et al., hep-ph/0412158, published as CERN Yellow Report, CERN2005-005, Geneva: CERN, 2005.

[26] S. Godfrey and N. Isgur, Phys. Rev. D 32, 189 (1985).

[27] S. Capstick and N. Isgur, Phys. Rev. D 34, 2809 (1986).

[28] S. Godfrey, Phys. Rev. D 70, 054017 (2004).

[29] D. Ebert, R. N. Faustov and V. O. Galkin, Phys. Rev. D 67, 014027 (2003), and references therein; Mod. Phys. Lett. A 20, 1887 (2005).

[30] E. Eichten, K. Gottfried, T. Kinoshita, K. D. Lane and T. M. Yan, Phys. Rev. D 17, 3090 (1978) [Erratum-ibid. D 21, 313 (1980)]; ibid.. D 21, 203 (1980).

[31] E. J. Eichten, K. Lane and C. Quigg, Phys. Rev. D 69, 094019 (2004); Phys. Rev. D 73, 014014 (2006) [Erratum-ibid. D 73, 079903 (2006)].

[32] A. De Rujula, H. Georgi and S. L. Glashow, Phys. Rev. D 12, 147 (1975).

[33] A. D. Sakharov, Sov. Phys. JETP 51, 1059 (1980) [Zh. Eksp. Teor. Fiz. 78, 2112 (1980)].

[34] S. Gasiorowicz and J. L. Rosner, Am. J. Phys. 49, 954 (1981).

[35] W.-M. Yao et al. [Particle Data Group], J. Phys. G 33, 1 (2006).

[36] R. Roncaglia, A. Dzierba, D. B. Lichtenberg and E. Predazzi, Phys. Rev. D 51, 1248 (1995), and references therein.

[37] M. Karliner and H. J. Lipkin, Phys. Lett. B 638, 221 (2006).

[38] R. L. Jaffe and F. Wilczek, Phys. Rev. Lett. 91, 232003 (2003).

[39] F. Wilczek, hep-ph/0409168, in From fields to strings: Circumnavigating theoretical physics: Ian Kogan memorial collection, edited by M. Shifman et al., vol. 1 (World Scientific, Singapore, 2005), p. 77; A. Selem, Senior Thesis, M. I. T., 2005 (unpublished); A. Selem and F. Wilczek, hep-ph/0602128, in Proc. Ringberg Workshop On New Trends In HERA Physics 2005, 2-7 October 2005, Tegernsee, Germany, edited by G. Grindhammer et al. (World Scientific, Hackensack, NJ, 2006), p. 337. 
[40] M. A. Shifman, A. I. Vainshtein and V. I. Zakharov, Nucl. Phys. B 147, 519 (1979); ibid. B 147, 385 (1979); ibid. B 147, 448 (1979).

[41] T. Schafer and E. V. Shuryak, Rev. Mod. Phys. 70, 323 (1998).

[42] F. J. Gilman, M. Kugler and S. Meshkov, Phys. Lett. B 45, 481 (1973); Phys. Rev. D 9, 715 (1974).

[43] F. J. Gilman and I. Karliner, Phys. Lett. B 46, 426 (1973); Phys. Rev. D 10, 2194 (1974).

[44] A. J. G. Hey and J. Weyers, Phys. Lett. B 48 (1974) 69.

[45] H. J. Melosh, Phys. Rev. D 9, 1095 (1974).

[46] L. Micu, Nucl. Phys. B 10, 521 (1969).

[47] E. W. Colglazier and J. L. Rosner, Nucl. Phys. B 27, 349 (1971).

[48] W. P. Petersen and J. L. Rosner, Phys. Rev. D 6 (1972) 820; ibid. D 7, 747 (1973).

[49] A. Le Yaouanc, L. Oliver, O. Pene and J. C. Raynal, Phys. Rev. D 8, 2223 (1973); ibid. D 9, 1415 (1974); ibid. D 11, 1272 (1975).

[50] R. P. Feynman, M. Kislinger and F. Ravndal, Phys. Rev. D 3, 2706 (1971).

[51] N. Isgur and G. Karl, Phys. Rev. D 18, 4187 (1978); ibid. Phys. Rev. D 19, 2653 (1979) [Erratum-ibid. D 23, 817 (1981)]; ibid. D 201191 (1979).

[52] E. van Beveren, et al., Z. Phys. C 30, 615 (1986).

[53] A. Dobado and J. R. Peláez, Phys. Rev. D 47, 4883 (1993); ibid. 56, 3057 (1997).

[54] R. L. Goble and J. L. Rosner, Phys. Rev. D 5, 2345 (1972); R. L. Goble, R. Rosenfeld and J. L. Rosner, Phys. Rev. D 39, 3264 (1989).

[55] I. Caprini, G. Colangelo, and H. Leutwyler, Phys. Rev. Lett. 96, 132001 (2006).

[56] E. van Beveren, D. V. Bugg, F. Kleefeld and G. Rupp, Phys. Lett. B 641, 265 (2006).

[57] M. Pennington, Phys. Rev. Lett. 97, 011601 (2006).

[58] E. M. Aitala et al. [E791 Collaboration], Phys. Rev. Lett. 86, 770 (2001); I. Bediaga, Braz. J. Phys. 34, 1398 (2004).

[59] M. Ablikim et al. [BES Collaboration], Phys. Lett. B 598, 149 (2004).

[60] D. V. Bugg, hep-ph/0608081.

[61] D. V. Bugg, hep-ex/0510014 
[62] J. Oller, Phys. Rev. D 71, 054030 (2005) and references therein; S. DescotesGenon and B. Moussallam, Eur. Phys. J. C 48, 553 (2006).

[63] E. M. Aitala et al. [E791 Collaboration], Phys. Rev. Lett. 89, 121801 (2002); Phys. Rev. D 73, 032004 (2006).

[64] M. Ablikim et al. [BES Collaboration], Phys. Lett. B 633, 681 (2006).

[65] D. V. Bugg, Phys. Lett. B 632, 471 (2006), and references therein.

[66] C. Cawlfield et al. [CLEO Collaboration], Phys. Rev. D 74, 031108(R) (2006).

[67] J. L. Rosner, Phys. Rev. D 74, 076006 (2006).

[68] B. Aubert et al. [BaBar Collaboration], Phys. Rev. Lett. 95, 121802 (2005).

[69] B. Aubert et al. [BaBar Collaboration], Phys. Rev. D 74, 032003 (2006).

[70] M. Chanowitz, Phys. Rev. Lett. 95, 172001 (2005).

[71] M. Campbell, M. S. Thesis, Univ. of Glasgow, 1997, as quoted in R. A. Briere et al., CLNS-01-1742.

[72] F. Ambrosino et al. [KLOE Collaboration], hep-ex/0612029. The method used to extract the gluon content of $\eta^{\prime}$ was suggested by J. Rosner, Phys. Rev. D 27, 1101 (1983).

[73] F. E. Close and Q. Zhao, Phys. Rev. D 71, 094022 (2005).

[74] D. Besson et al. [CLEO Collaboration], hep-ex/0512003, submitted to Phys. Rev. D.

[75] C. McNeile, C. Michael and P. Pennanen [UKQCD Collaboration], Phys. Rev. D 65, 094505 (2002); C. Michael, hep-ph/0308293; C. McNeile and C. Michael [UKQCD Collaboration], Phys. Rev. D 73, 074506 (2006); T. Burns and F. E. Close, Phys. Rev. D 74, 034003 (2006).

[76] J. N. Hedditch et al., Phys. Rev. D 72, 114507 (2005)

[77] G. S. Adams et al. [E852 Collaboration], Phys. Rev. Lett. 81, 5760 (1998).

[78] A. R. Dzierba et al., Phys. Rev. D 73, 072001 (2006).

[79] C. Meyer, presented at the Conference on the Intersections of Particle and $\mathrm{Nu}$ clear Physics (CIPANP 2006), Rio Grande, Puerto Rico, May 30 - June 3, 2006, AIP Conf. Proc. 870, edited by T. M. Liss, p. 380.

[80] C. Michael, hep-lat/0609008, presented at 4th International Conference On Quarks And Nuclear Physics (QNP06), 5-10 June 2006, Madrid, Spain.

[81] M. K. Gaillard, B. W. Lee and J. L. Rosner, Rev. Mod. Phys. 47, 277 (1975). 
[82] B. Aubert et al. [BaBar Collaboration], Phys. Rev. Lett. 97, 232001 (2006).

[83] J. L. Rosner, Phys. Rev. D 52, 6461 (1995).

[84] M. Artuso et al. [CLEO Collaboration], Phys. Rev. Lett. 86, 4479 (2001).

[85] B. Aubert et al. [BaBar Collaboration], Phys. Rev. Lett. 98, 012001 (2007).

[86] K. Abe et al. [Belle Collaboration], Belle report BELLE-CONF-0602, hep-ex/0608043, submitted to ICHEP 06 (op. cit.)

[87] R. Mizuk et al. [Belle Collaboration], Phys. Rev. Lett. 94, 122002 (2005).

[88] R. Chistov et al. [Belle Collaboration], Phys. Rev. Lett. 97, 162001 (2006).

[89] B. Aubert et al. [BaBar Collaboration], SLAC-PUB-11980, BABAR-CONF-0601, hep-ex/0607042, submitted to ICHEP 06.

[90] B. Aubert et al. [BaBar Collaboration], Phys. Rev. Lett. 90, 242001 (2003); D. Besson et al. [CLEO Collaboration], Phys. Rev. D 68, 032002 (2003); K. Abe et al. [Belle Collaboration], Phys. Rev. Lett. 92, 012002 (2004).

[91] H. Marsiske, at Flavor Physics and CP Violation Conference, Vancouver, BC, April, 2006.

[92] B. Aubert et al. [BaBar Collaboration], Phys. Rev. D 74, 031103 (2006); S. J. Gowdy [for the BaBar Collaboration], at Moriond 2006 (QCD and Hadronic Interactions at High Energy), hep-ex/0605086.

[93] E. van Beveren and G. Rupp, Phys. Rev. Lett. 91, 012003 (2003); Eur. Phys. J. C 32, 493 (2004).

[94] F. E. Close, Int. J. Mod. Phys. A 20, 5156 (2005).

[95] K. Abe et al. [Belle Collaboration], Belle report BELLE-CONF-0643, hep-ex/0608031, submitted to ICHEP 06 (op. cit.)

[96] A. Martin, Phys. Lett. B 100, 511 (1981).

[97] B. Zhang, X. Liu, W. Z. Deng and S. L. Zhu, hep-ph/0609013.

[98] B. Aubert et al. [BaBar Collaboration], Phys. Rev. Lett. 97, 222001 (2006).

[99] E. van Beveren and G. Rupp, Phys. Rev. Lett. 97, 202001 (2006).

[100] F. E. Close, C. E. Thomas, O. Lakhina and E. S. Swanson, hep-ph/0608139.

[101] P. Colangelo, F. De Fazio and S. Nicotri, Phys. Lett. B 642, 48 (2006).

[102] S. Anderson et al. [CLEO Collaboration], Nucl. Phys. A 663, 647 (2000).

[103] K. Abe et al. [Belle Collaboration], Phys. Rev. D 69, 112002 (2004). 
[104] J. M. Link et al. [FOCUS Collaboration], Phys. Lett. B 586, 11 (2004).

[105] P. Colangelo, F. De Fazio, R. Ferrandes and S. Nicotri, hep-ph/0609240.

[106] M. Artuso al. [CLEO Collaboration], Phys. Rev. Lett. 95, 251801 (2005).

[107] C. Aubin et al., Phys. Rev. Lett. 95, 122002 (2006).

[108] B. Aubert et al. [BaBar Collaboration], hep-ex/0607094.

[109] S. Stone [CLEO Collaboration], presented at ICHEP 06 (op. cit.), http://ichep06.jinr.ru/reports/179_10s2_18p05_Stone.pdf, hep-ex/0610026.

[110] A. Abulencia et al. [CDF Collaboration], Phys. Rev. Lett. 97, 242003 (2006).

[111] M. Okamoto, PoS LAT2005, 013 (2006), hep-lat/0510113.

[112] J. L. Rosner, Phys. Rev. D 42, 3732 (1990).

[113] D. Acosta et al. [CDF Collaboration], Phys. Rev. Lett. 96, 202001 (2006).

[114] I. V. Gorelov [for the CDF Collaboration], talk given at the Second Meeting of the APS Topical Group on Hadron Physics (GHP 2006), October 2224, Nashville, Tennessee, Proceedings to be published by Institute of Physics, hep-ex/0701056.

[115] E. Jenkins, Phys. Rev. D 54, 4515 (1996); ibid. 55, 10 (1997).

[116] M. Karliner and H. J. Lipkin, hep-ph/0307243 (unpublished).

[117] J. L. Rosner, Phys. Rev. D 75, 013009 (2007).

[118] M. Aoki [for the CDF Collaboration], presented to Quarkonium Working Group, Brookhaven Natl. Lab., June 27-30, 2006, updating W. Wester, Nucl. Phys. B Proc. Suppl. 156, 240 (2006); D. Acosta et al., Phys. Rev. Lett. 96, 082002 (2006).

[119] I. F. Allison, C. T. H. Davies, A. Gray, A. S. Kronfeld, P. B. Mackenzie and J. N. Simone [HPQCD Collaboration], Phys. Rev. Lett. 94, 172001 (2005).

[120] V. M. Abazov et al. [D0 Collaboration], Phys. Rev. Lett. 97, 021802 (2006).

[121] Preliminary values quoted by K. Ikado et al. [Belle Collaboration], hep-ex/0604018; hep-ex/0605068, have been corrected for revised estimates of efficiency. See Belle report BELLE-CONF-0671, reported by T. Browder at ICHEP 06 (op. cit.)

[122] Periodic updates may be found at http://www.slac.stanford.edu/xorg/hfag/.

[123] A. Gray et al. [HPQCD Collaboration], Phys. Rev. Lett. 95, 212001 (2005). 
[124] A. Abulencia et al. [CDF Collaboration], Fermilab report FERMILAB-PUB06-321-E, hep-ex/0609021, submitted to Phys. Rev. Letters.

[125] J. L. Rosner et al. [CLEO Collaboration], Phys. Rev. Lett. 95, 102003 (2005).

[126] P. Rubin et al. [CLEO Collaboration], Phys. Rev. D 72, 092005 (2005).

[127] T. Manke et al. [CP-PACS Collaboration], Phys. Rev. D 62, 114508 (2000); M. Okamoto et al. [CP-PACS Collaboration], ibid. 65, 095408 (2002).

[128] M. Andreotti et al. [Fermilab E835 Collab.], Phys. Rev. D 72, 032001 (2005).

[129] J. Stubbe and A. Martin, Phys. Lett. B 271, 208 (1991).

[130] J. L. Rosner, Ann. Phys. (N.Y.) 319, 1 (2005).

[131] D. Besson et al. [CLEO Collaboration], Phys. Rev. Lett. 96, 092002 (2006).

[132] Q. He et al. [CLEO Collaboration], Phys. Rev. Lett. 95, 121801 (2005) [Erratum-ibid. 96, 199903 (2006)].

[133] M. Ablikim et al. [BES Collaboration], Phys. Lett. B 641, 145 (2006); Phys. Rev. Lett. 97, 121801 (2006); hep-ex/0612056.

[134] M. Ablikim et al. [BES Collaboration], paper no. 123, 22nd International Symposium on Lepton-Photon Interactions at High Energy (LP 2005), Uppsala, Sweden, 30 June - 5 July 2005.

[135] N. E. Adam [CLEO Collaboration], Phys. Rev. Lett. 96, 082004 (2006).

[136] J. Z. Bai et al. [BES Collaboration], Phys. Lett. B 605, 63 (2005).

[137] T. E. Coan et al. [CLEO Collaboration], Phys. Rev. Lett. 96, 182002 (2006).

[138] R. A. Briere et al. [CLEO Collaboration], Phys. Rev. D 74, 031106 (2006).

[139] G. S. Adams et al. [CLEO Collaboration], Phys. Rev. D 73, 012002 (2006).

[140] G. S. Huang et al., CLEO Report CLEO-CONF 05-13, paper no. LP-2005-443, LP 2005, op. cit.

[141] S. Godfrey, invited talk at FPCP 2006 (Flavor Physics and CP Violation, Vancouver, BC, April 2006), hep-ph/0605152.

[142] E. Swanson, CIPANP 2006, op. cit., p. 349.

[143] S. K. Choi et al. [Belle Collaboration], Phys. Rev. Lett. 91, 262001 (2003).

[144] B. Aubert et al. [BaBar Collaboration], Phys. Rev. D 71, 071103 (2005).

[145] D. Acosta et al. [CDF II Collaboration], Phys. Rev. Lett. 93, 072001 (2004); V. M. Abazov et al. [D0 Collaboration], Phys. Rev. Lett. 93, 162002 (2004). 
[146] F. E. Close and P. R. Page, Phys. Lett. B 578, 119 (2004); N. A. Tornqvist, hep-ph/0308277; E. S. Swanson, Phys. Lett. B 588, 189 (2004); ibid. 598, 197 (2004).

[147] J. L. Rosner, Phys. Rev. D 70, 094023 (2004).

[148] K. Abe et al., Belle report BELLE-CONF-0541, hep-ex/0505038, paper no. LP-2005-176, LP 2005, op. cit.

[149] A. Abulencia et al. [CDF Collaboration], hep-ex/0612053.

[150] G. Gokhroo et al. [Belle Collaboration], Phys. Rev. Lett. 97, 162002 (2006).

[151] K. Abe et al., Belle report BELLE-CONF-0540, hep-ex/0505037, paper no. LP-2005-175, LP 2005, op. cit.

[152] B. Aubert et al. [BaBar Collaboration], Phys. Rev. Lett. 96. 052002 (1996).

[153] K. Abe et al. [Belle Collaboration], Phys. Rev. Lett. 96. 082003 (2006).

[154] K. Abe et al. [Belle Collaboration], hep-ex/0507019, submitted to Phys. Rev. Letters; L. Hinz, CIPANP 2006, op. cit., p. 345.

[155] K. Abe et al. [Belle Collaboration], Phys. Rev. Lett. 94, 182002 (2005).

[156] H. Severini et al. [CLEO Collaboration], Phys. Rev. Lett. 92, 222002 (2004).

[157] B. Aubert et al. [BaBar Collaboration], Phys. Rev. Lett. 95, 142001 (2005).

[158] F. J. Llanes-Estrada, Phys. Rev. D 72, 031503 (2005).

[159] L. Maiani, V. Riquer, F. Piccinini and A. D. Polosa, Phys. Rev. D 72, 031502 (2005).

[160] S. L. Zhu, Phys. Lett. B 625, 212 (2005); E. Kou and O. Pene, Phys. Lett. B 631, 164 (2005); F. E. Close and P. R. Page, Phys. Lett. B 628, 215 (2005).

[161] T. E. Coan et al. [CLEO Collaboration], Phys. Rev. Lett. 96, 162003 (2006).

[162] CLEO Collaboration, reported by S. Blusk, CIPANP 2006, op. cit., p. 341.

[163] F. E. Close and P. R. Page, Phys. Lett. B 628, 215 (2005).

[164] G. Peter Lepage, Ann. Phys. 315, 193 (2005).

[165] D. Besson et al. [CLEO Collaboration], Phys. Rev. D 74, 012003 (2006).

[166] C. Cawlfield et al. [CLEO Collaboration], Phys. Rev. D 73, 012003 (2006).

[167] G. Tatishvili, CIPANP 2006, op. cit., p. 356.

[168] B. Aubert et al. [BaBar Collaboration], Phys. Rev. Lett. 96, 232001 (2006). 
[169] K. Abe et al. [Belle Collaboration], hep-ex/0512034, contributed to LP 2005 (op. cit.), and to EPS International Europhysics Conference on High Energy Physics (HEP-EPS 2005), Lisbon, Portugal.

[170] G. S. Adams et al. [CLEO Collaboration], Phys. Rev. Lett. 94, 012001 (2005).

[171] M. Artuso et al. [CLEO Collaboration], Phys. Rev. Lett. 94, 032001 (2005).

[172] W. Kwong and J. L. Rosner, Phys. Rev. D 38, 3179 (1988).

[173] P. Moxhay and J. L. Rosner, Phys. Rev. D 28, 1132 (1983); R. McClary and N. Byers, ibid. 28, 1692 (1983). See also T. Skwarnicki, hep-ex/0505050, at 40th Rencontres de Moriond On QCD and High Energy Hadronic Interactions, 12-19 Mar 2005, La Thuile, Aosta Valley, Italy.

[174] S. Godfrey and J. L. Rosner, Phys. Rev. D 64, 074011 (2001) [Erratum-ibid. D 65, $039901(2002)]$.

[175] S. Godfrey and J. L. Rosner, Phys. Rev. D 66, 014012 (2002).

[176] M. B. Voloshin, Mod. Phys. Lett. A 19, 2895 (2004).

[177] I. C. Brock et al. [CLEO Collaboration], Phys. Rev. D 43, 1448 (1991). 